\title{
Incompressible limits of lattice Boltzmann equations using multiple relaxation times
}

\author{
Paul J. Dellar \\ OCIAM, Mathematical Institute, 24-29 St Giles', Oxford, OX1 3LB, United Kingdom \\ E-mail: pdellar@na-net.ornl.gov \\ Submitted 22nd September 2002, accepted 12th May 2003 by J. Comput. Phys.
}

\begin{abstract}
Lattice Boltzmann equations using multiple relaxation times are intended to be more stable than those using a single relaxation time. The additional relaxation times may be adjusted to suppress non-hydrodynamic modes that do not appear directly in the continuum equations, but may contribute to instabilities on the grid scale. If these relaxation times are fixed in lattice units, as in previous work, solutions computed on a given lattice are found to diverge in the incompressible (small Mach number) limit. This non-existence of an incompressible limit is analysed for an inclined one dimensional jet. An incompressible limit does exist if the nonhydrodynamic relaxation times are not fixed, but scaled by the Mach number in the same way as the hydrodynamic relaxation time that determines the viscosity.
\end{abstract}

\section{INTRODUCTION}

Methods based on lattice Boltzmann equations (LBE) are a promising alternative to conventional numerical methods for simulating fluid flows $[9,32]$. Using a velocity-space truncation of the Boltzmann equation from the kinetic theory of gases $[6,7,18]$, lattice Boltzmann methods lead to linear, constant coefficient hyperbolic systems with nonlinear source terms. Almost all lattice Boltzmann equations simulate compressible fluids with some finite sound speed $c_{\mathrm{s}}$. However, the computed solutions are expected to converge towards an incompressible limit when the fluid speed $|\mathbf{u}|$ is sufficiently small compared with $c_{\mathrm{s}}$, i.e. as the Mach number $\mathrm{Ma}=|\mathbf{u}| / c_{\mathrm{s}}$ tends to zero. Most recent work with lattice Boltzmann equations follows Chen et al. [8] and Qian et al. [29] in employing the Bhatnagar-Gross-Krook (BGK) collision operator [5], for which every variable relaxes towards equilibrium with the same timescale $\tau$. The BGK approximation was originally seen as a simplification over previous lattice Boltzmann equations using first linearized forms of binary collision operators originating in lattice gas cellular automata, and then general linear operators constrained by symmetry and conservation properties [4, 23, 24]. These historical developments have recently been reviewed by Succi et al. [33]. Lallemand and Luo [25] found that some more complicated collision operators improve stability at high Reynolds numbers compared with the BGK collision operator. In this paper we show that incompressible limits do not exist for lattice Boltzmann equations with these collision operators, unless they are modified to make every timescale proportional to the Mach number.

The Boltzmann equation for a discrete velocity space with the BGK collision operator may be written as

$$
\partial_{t} f_{i}+\boldsymbol{\xi}_{i} \cdot \nabla f_{i}=-\frac{1}{\tau}\left(f_{i}-f_{i}^{(0)}\right)
$$

where the distribution functions $f_{i}$, equilibrium $f_{i}^{(0)}$, lattice vectors $\boldsymbol{\xi}_{i}$, and other variables are defined in detail below. For suitable choices of the $\boldsymbol{\xi}_{i}$ and $f_{i}^{(0)}$, solutions of (1) may be shown to simulate the Navier-Stokes equations with kinematic viscosity $\nu$ proportional to $\tau$. Equation (1) is sometimes called a discrete Boltzmann equation. It is usually implemented computationally as the fully discrete system, or lattice Boltzmann equation,

$$
\bar{f}_{i}\left(\mathbf{x}+\boldsymbol{\xi}_{i} \Delta t, t+\Delta t\right)-\bar{f}_{i}(\mathbf{x}, t)=-\frac{\Delta t}{\tau+\Delta t / 2}\left(\bar{f}_{i}(\mathbf{x}, t)-f_{i}^{(0)}(\mathbf{x}, t)\right)
$$


for the modified distribution functions $\bar{f}_{i}$ defined below, which is a second order accurate approximation to (1) in both space and time. For spatially uniform solutions, (2) may be rearranged into the form

$$
\left(\bar{f}_{i}(t+\Delta t)-f_{i}^{(0)}\right)=-\left(\frac{1-2 \tau / \Delta t}{1+2 \tau / \Delta t}\right)\left(\bar{f}_{i}(t)-f_{i}^{(0)}\right) .
$$

The scheme (2) is typically used with $\tau \ll \Delta t$ to attain high grid Reynolds numbers, for which the coefficient $\gamma=-(1-$ $2 \tau / \Delta t) /(1+2 \tau / \Delta t)$ in (3) is close to -1 . In other words, for small $\tau$ the discrete variables $\bar{f}_{i}$ are over relaxed by an amount close to the linear stability boundary, rather than driven rapidly towards equilibrium as in the continuum system (1). In other words, the non-equilibrium parts of the distribution functions are rapidly oscillating but only slowly decaying. This is the source of the instabilities that restrict the maximum feasible Reynolds number for a given lattice.

To ensure isotropy, most lattice Boltzmann equations include more variables than appear in the hydrodynamic equations that they simulate. For example, the most common two dimensional lattice Boltzmann equation [29] includes nine distribution functions, while only six independent variables are necessary to recover the two dimensional Navier-Stokes equations. These six variables are the scalar density $\rho$, the velocity $\mathbf{u}$, and the symmetric momentum flux tensor $\boldsymbol{\Pi}$. The three extra variables are associated with non-hydrodynamic or "ghost" variables $[3,4,12]$ that have no effect on the intended hydrodynamic behavior at large spatial scales, but may dominate at the smallest permitted scales comparable with the computational lattice [12].

Lallemand and Luo [25] proposed using a more complicated collision operator that over relaxes only those combinations of the $f_{i}$ that contribute to the momentum flux $\Pi$, and hence to the viscous stress, while damping the three non-hydrodynamic combinations that do not appear in the Navier-Stokes equations. This modification might be expected to improve stability for a given Reynolds number, and was extended to three dimensions by d'Humières et al. [14]. Lallemand and Luo [25] used a non-hydrodynamic relaxation time $\tau_{g}$ slightly larger that $\Delta t / 2$, while Higuera et al. [24] and Succi [32] recommended choosing $\tau_{g}=\Delta t / 2$, for which $\gamma=0$ in (3), to maximally damp the non-hydrodynamic variables. The latter is equivalent to McNamara et al.'s [27] approach of setting the non-hydrodynamic modes to zero at each lattice point after each timestep.

The potential gains available from using a multiple relaxation time (MRT) collision operator to damp non-hydrodynamic modes are illustrated by the solutions shown in Fig. 1. The four subplots show the results of simulating the roll-up of two antiparallel shear layers through a Kelvin-Helmholtz instability, as considered by Minion and Brown [28] (see Section 5). The initial conditions were given by equation (32) below with $\kappa=80, \delta=0.05$. The Reynolds number was $\operatorname{Re}=30000$, and the Mach number was $\mathrm{Ma}=\sqrt{3} / 25 \approx 0.07$. Using the BGK collision operator, the simulation on a $128^{2}$ lattice becomes unstable and "blows up" before $t=1.0$. On a $256^{2}$ lattice the BGK simulation remains stable, but develops two spurious vortices of the kind investigated by Minion and Brown [28]. By contrast, the simulation on a coarse $128^{2}$ grid using a multiple relaxation time collision operator with $\tau_{\mathrm{g}}=\Delta t / 2$ compares favorably with the well resolved $512^{2}$ solution that uses the BGK collision operator. Although the shear layers have been thickened by the coarse grid, the $128^{2}$ MRT solution is stable and lacks spurious vorticies. Similarly, Dellar [11] found that enhancing the bulk viscosity while leaving the non-hydrodynamic modes unchanged could suppress spurious vortex formation.

In this paper we show that solutions computed by lattice Boltzmann equations that damp non-hydrodynamic modes in this way, with timescales that are fixed multiples of $\Delta t$, do not converge to an incompressible limit as $\mathrm{Ma} \rightarrow 0$. Instead, the solutions on a fixed lattice diverge as $O\left(\mathrm{Ma}^{-1}\right)$ in the small Mach number limit. This observation, originally based on numerical experiments, is confirmed by theoretical analysis of a linearized problem. It applies not just to the usual isothermal lattice Boltzmann equation, but also to an "incompressible" modification [35, 20], because the two equations coincide when linearized around a uniform rest state. The difficulty arises from the use of non-hydrodynamic relaxation times that are fixed in lattice units, as employed by Lallemand and Luo [25] and d'Humières et al. [14], and may be avoided by scaling every relaxation time with the Mach number in the same way as the stress relaxation time, $\tau_{\Pi}=\sqrt{3} \mathrm{~N} \mathrm{Ma} / \mathrm{Re}$ in lattice units. In outline, the details being given in Section 7, a lattice Boltzmann scheme requires $O\left(\mathrm{Ma}^{-1}\right)$ timesteps to reach a fixed macroscopic time, as determined in terms of an eddy turnover time for instance. Thus the eigenmodes of the linearized system decay in proportion to $(1-O(\tau))^{1 / \mathrm{Ma}}$, and this expression attains a nonzero limit only if $\tau=O(\mathrm{Ma})$ as $\mathrm{Ma} \rightarrow 0$. In other words, the correct incompressible limit exists if the non-hydrodynamic modes are assigned a fixed Reynolds number $R_{\mathrm{g}}$, instead of an explicit relaxation time. This Reynolds number may differ from the usual hydrodynamic Reynolds number related to the shear viscosity, but determines the non-hydrodynamic relaxation time as a function of Reynolds number, Mach number, and spatial resolution by the same formula that relates the stress relaxation time to the usual Reynolds number.

The above all applies to a fixed lattice. The divergence at small Mach numbers may be suppressed by suitably refining the lattice as the Mach number decreases. The error is proportional to $\mathrm{Ma}^{-1} N^{-3}$, where $N$ is the number of lattice points per unit interval, and so may be made small by increasing $N$. However, this soon becomes very expensive because the computational work is proportional to $N^{(D+1)} \mathrm{Ma}^{-1}$ in $D$ spatial dimensions. Thus it is common to test a scheme by separately verifying 

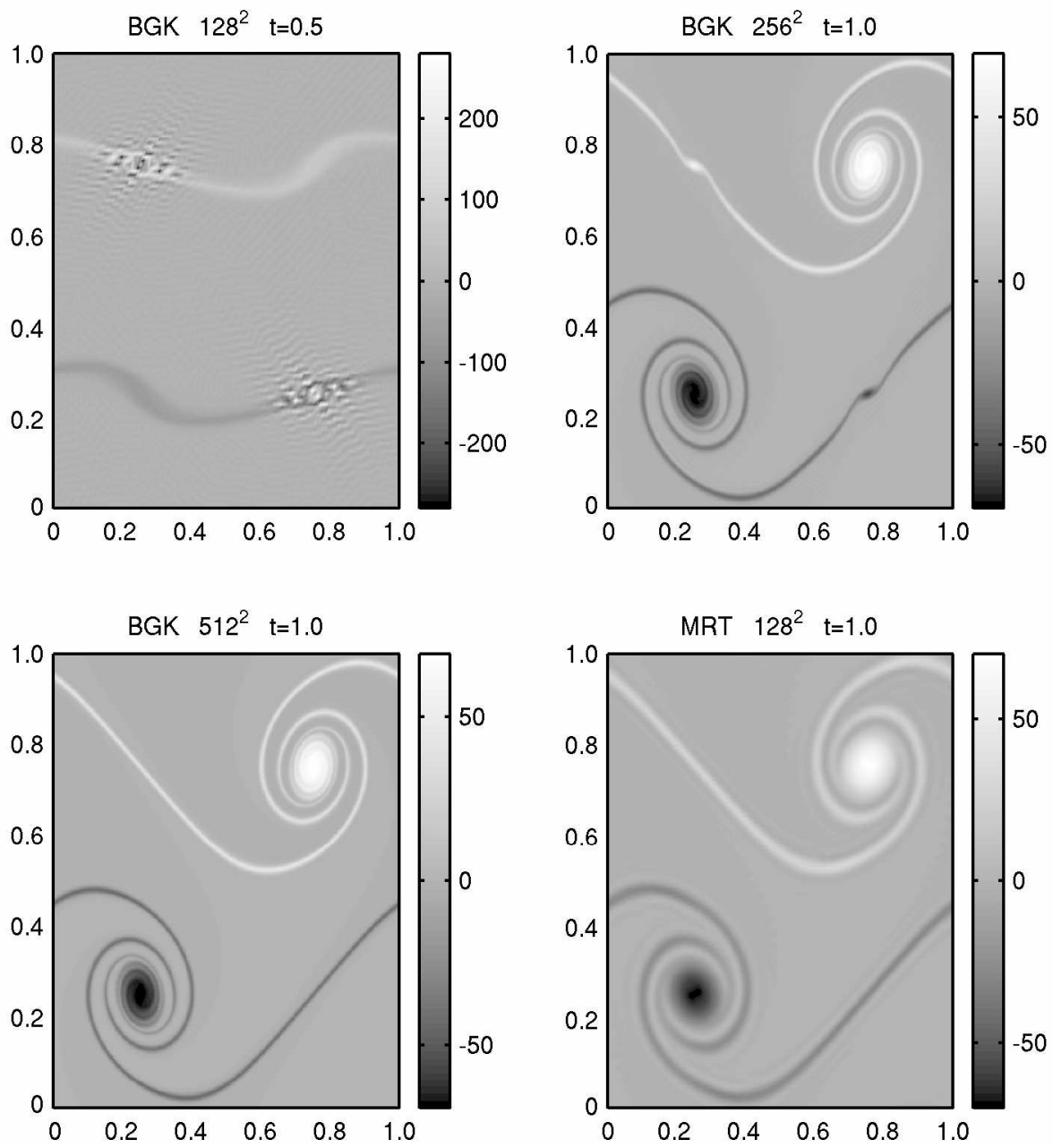

FIG. 1. Vorticity during the roll up of a perturbed doubly-periodic shear layer at $R e=30000$. For this high value of the Reynolds number, the BGK simulation on a $128^{2}$ grid develops grid scale instabilities leading to "blow up" before $t=1.0$, while the BGK simulation on a $256^{2}$ grid forms spurious vortices of the kind investigated by Minion and Brown [28]. The $128^{2}$ grid simulation using a multiple relaxation time (MRT) collision operator with $\tau_{\mathrm{g}}=\Delta t / 2$ compares more favorably with the $512^{2}$ grid BGK simulation. Although the shear layers have been thickened by the coarse grid, this $128^{2}$ MRT simulation is stable and lacks spurious vorticies. 
spatial convergence at fixed Mach number, and Mach number convergence on a fixed lattice; except the latter limit does not exist for the proposed MRT lattice Boltzmann equations.

\section{LATTICE BOLTZMANN HYDRODYNAMICS}

In the lattice Boltzmann approach to hydrodynamics, macroscopic variables like the fluid density $\rho$ and velocity $\mathbf{u}$ are expressed as moments of a discrete set of distribution functions $f_{i}(\mathbf{x}, t)$,

$$
\rho=\sum_{i=0}^{n} f_{i}, \quad \rho \mathbf{u}=\sum_{i=0}^{n} \boldsymbol{\xi}_{i} f_{i}, \quad \mathbf{\Pi}=\sum_{i=0}^{n} \boldsymbol{\xi}_{i} \boldsymbol{\xi}_{i} f_{i},
$$

where $\boldsymbol{\xi}_{0}, \ldots, \boldsymbol{\xi}_{n}$ are a discrete set of particle velocities associated with the $f_{i}$.

These distribution functions evolve according to the discrete Boltzmann equation,

$$
\partial_{t} f_{i}+\boldsymbol{\xi}_{i} \cdot \nabla f_{i}=-\Omega_{i j}\left(f_{j}-f_{j}^{(0)}\right), \text { for } i=0, \ldots, n,
$$

with an implied summation over the repeated index $j$. The collision matrix $\Omega_{i j}$ and equilibrium distributions $f_{j}^{(0)}$ must be chosen so as to recover Navier-Stokes behavior for the macroscopic variables in a slowly varying limit. In particular, the right hand side of (5) should conserve mass and momentum, in the sense that $[4,33]$

$$
\sum_{i=0}^{n} \Omega_{i j}\left(f_{j}-f_{j}^{(0)}\right)=0, \quad \sum_{i=0}^{n} \boldsymbol{\xi}_{i} \Omega_{i j}\left(f_{j}-f_{j}^{(0)}\right)=0 .
$$

Moreover, $\Omega_{i j}$ should only depend on the angle between the two particle velocities $\boldsymbol{\xi}_{i}$ and $\boldsymbol{\xi}_{j}$ to ensure isotropy [32, 4, 24, 16]. The commonly employed Bhatnagar-Gross-Krook (BGK) approximation [5] takes

$$
\Omega_{i j}=\frac{1}{\tau} \delta_{i j}
$$

so that every $f_{i}$ relaxes towards its equilibrium value $f_{i}^{(0)}$ with the same timescale $\tau$.

The Chapman-Enskog expansion [18, 7, 34] seeks slowly varying solutions to (5) by inserting a formal parameter $1 / \epsilon$ in front of the collision operator right hand side,

$$
\partial_{t} f_{i}+\boldsymbol{\xi}_{i} \cdot \nabla f_{i}=-\frac{1}{\epsilon} \Omega_{i j}\left(f_{j}-f_{j}^{(0)}\right), \text { for } i=0, \ldots, n,
$$

so that the slowly varying limit corresponds to $\epsilon \rightarrow 0$. The Chapman-Enskog expansion is a multiple scales expansion of both $f$ and $t$, but not $\mathbf{x}$, in powers of $\epsilon$,

$$
f_{i}=f_{i}^{(0)}+\epsilon f_{i}^{(1)}+\epsilon^{2} f_{i}^{(2)}+\cdots, \quad \partial_{t}=\partial_{t_{0}}+\epsilon \partial_{t_{1}}+\cdots,
$$

subject to the solvability conditions

$$
\sum_{i=0}^{n} f_{i}^{(m)}=\sum_{i=0}^{n} \boldsymbol{\xi}_{i} f_{i}^{(m)}=0, \text { for } m=1,2, \ldots
$$

Substituting the expansions (9) into (5), collecting terms at each order, and then taking moments we obtain macroscopic mass and momentum conservation equations in the form

$$
\partial_{t} \rho+\nabla \cdot(\rho \mathbf{u})=0, \quad \partial_{t}(\rho \mathbf{u})+\nabla \cdot\left(\boldsymbol{\Pi}^{(0)}+\epsilon \boldsymbol{\Pi}^{(1)}+\cdots\right)=0
$$

where $\boldsymbol{\Pi}^{(n)}=\sum_{i=0}^{n} \boldsymbol{\xi}_{i} \boldsymbol{\xi}_{i} f_{i}^{(n)}$. The right hand sides vanish in (11), and $\rho$ and $\mathbf{u}$ require no superscripts, by virtue of the solvability conditions in (10).

To reproduce the compressible Euler equations, the first few moments of the equilibria $f_{i}^{(0)}$ must be

$$
\sum_{i=0}^{n} f_{i}^{(0)}=\rho, \quad \sum_{i=0}^{n} \boldsymbol{\xi}_{i} f_{i}^{(0)}=\rho \mathbf{u}, \quad \Pi^{(0)}=\sum_{i=0}^{n} \boldsymbol{\xi}_{i} \boldsymbol{\xi}_{i} f_{i}^{(0)}=\theta \rho \mathbf{l}+\rho \mathbf{u u},
$$




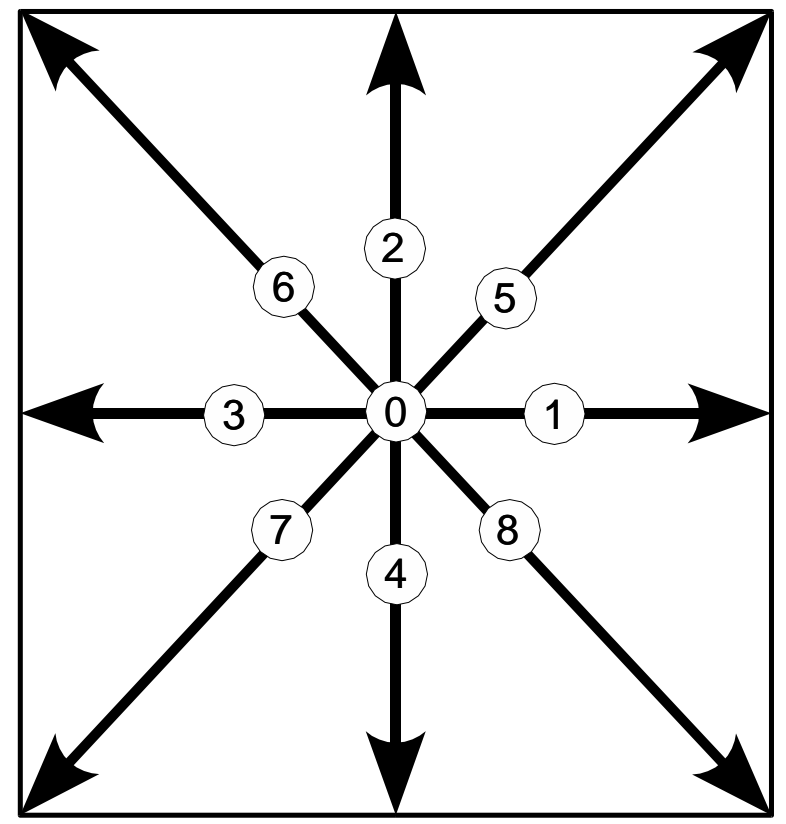

FIG. 2. The nine particle velocities $\boldsymbol{\xi}_{i}$ in the 2 D square lattice. In lattice units $\left|\boldsymbol{\xi}_{1}\right|=1$, and $\left|\boldsymbol{\xi}_{5}\right|=\sqrt{2}$.

where I denotes the identity tensor. The equation of state is thus $p=\theta \rho$, where $p$ is the pressure and $\theta$ the temperature. The most common lattice Boltzmann equation simulates an isothermal (constant $\theta$ ) fluid by using nine particle velocities arranged on a square lattice in two dimensions, as illustrated in Fig. 2. The equilibrium distributions are given by [9, 29, 21]

$$
f_{i}^{(0)}=w_{i} \rho\left(1+3 \boldsymbol{\xi}_{i} \cdot \mathbf{u}+\frac{9}{2}\left(\boldsymbol{\xi}_{i} \cdot \mathbf{u}\right)^{2}-\frac{3}{2} \mathbf{u}^{2}\right)
$$

in units where the (constant) temperature $\theta=1 / 3$, and the components of the particle speeds $\boldsymbol{\xi}_{i}$ take the integer values $\{-1,0,1\}$. The weight factors $w_{i}$ are

$$
w_{i}= \begin{cases}4 / 9, & \mathrm{i}=0 \\ 1 / 9, & \mathrm{i}=1,2,3,4 \\ 1 / 36, & \mathrm{i}=5,6,7,8\end{cases}
$$

The Navier-Stokes viscous stress is determined by $\Pi^{(1)}$, which may be evaluated from the evolution equation for $\Pi$,

$$
\partial_{t} \boldsymbol{\Pi}+\nabla \cdot\left(\sum_{i=0}^{n} \boldsymbol{\xi}_{i} \boldsymbol{\xi}_{i} \boldsymbol{\xi}_{i} f_{i}\right)=-\frac{1}{\epsilon} \sum_{i=0}^{n} \boldsymbol{\xi}_{i} \boldsymbol{\xi}_{i} \Omega_{i j}\left(f_{j}-f_{j}^{(0)}\right),
$$

obtained by applying $\sum_{i=0}^{n} \boldsymbol{\xi}_{i} \boldsymbol{\xi}_{i}$ to (5). At leading order in $\epsilon$ this becomes

$$
\partial_{t_{0}} \boldsymbol{\Pi}^{(0)}+\nabla \cdot\left(\sum_{i=0}^{n} \boldsymbol{\xi}_{i} \boldsymbol{\xi}_{i} \boldsymbol{\xi}_{i} f_{i}^{(0)}\right)=-\sum_{i=0}^{n} \boldsymbol{\xi}_{i} \boldsymbol{\xi}_{i} \Omega_{i j} f_{j}^{(1)}
$$

The multiple scales expansion of the time derivative in (9) enables us to replace $\partial_{t} \Pi^{(0)}$ by $\partial_{t_{0}} \boldsymbol{\Pi}^{(0)}$ to sufficient accuracy, and the latter expression may be evaluated in terms of the known quantities $\partial_{t_{0}} \rho$ and $\partial_{t_{0}}(\rho \mathbf{u})$ computed from the leading order terms in (11). The left hand side of (16) then simplifies to $-\theta \rho\left[\nabla \mathbf{u}+(\nabla \mathbf{u})^{\top}\right]$, which is a Newtonian viscous stress. Thus the collision matrix $\Omega_{i j}$ must be constrained so that the right hand side of (16) simplifies to $-\tau_{\Pi}^{-1} \sum_{i=0}^{n} \boldsymbol{\xi}_{i} \boldsymbol{\xi}_{i} f_{j}^{(1)}=-\tau_{\Pi}^{-1} \Pi^{(1)}$. The dynamic viscosity $\mu=\rho \nu$ is related to the timescale $\tau_{\Pi}$ by $\mu=\tau_{\Pi} \theta \rho$. 


\subsection{Incompressible lattice Boltzmann model}

The most common lattice Boltzmann equation, with equilibria given by (13), solves the compressible, isothermal NavierStokes equations in the form

$$
\begin{aligned}
\partial_{t} \rho+\nabla \cdot(\rho \mathbf{u}) & =0, \\
\partial_{t}(\rho \mathbf{u})+\nabla \cdot\left(\rho \mathbf{u u}+c_{\mathrm{s}}^{2} \rho \mathbf{l}\right) & =\nabla \cdot \mathrm{S}+O\left(\mathrm{Ma}^{3} / \mathrm{Re}\right) .
\end{aligned}
$$

The equation of state is $p=c_{\mathrm{s}}^{2} \rho$, with constant sound speed $c_{\mathrm{s}}=\theta^{1 / 2}$. The viscous stress $\mathbf{S}=\mu\left[\nabla \mathbf{u}+(\nabla \mathbf{u})^{\top}\right]$ is Newtonian, with shear viscosity $\mu$ and a nonzero bulk viscosity [11]. At low Mach numbers, Ma $=|\mathbf{u}| / c_{\mathrm{s}} \ll 1$, solutions of (17) approximate solutions of the incompressible ( $\rho=\rho_{0}$ is constant) Navier-Stokes equations with error $O\left(\mathrm{Ma}^{2}\right)$.

Zou et al. [35] and He and Luo [20] proposed the alternative equilibria

$$
f_{i}^{(0)}=w_{i}\left[\rho+\rho_{0}\left(3 \boldsymbol{\xi}_{i} \cdot \mathbf{u}+\frac{9}{2}\left(\boldsymbol{\xi}_{i} \cdot \mathbf{u}\right)^{2}-\frac{3}{2} \mathbf{u}^{2}\right)\right],
$$

for which solutions of the lattice Boltzmann equation approximate the macroscopic equations [20]

$$
\begin{aligned}
c_{\mathrm{s}}^{-2} \partial_{t} P+\nabla \cdot \mathbf{u} & =0 \\
\partial_{t} \mathbf{u}+\mathbf{u} \cdot \nabla \mathbf{u} & =-\nabla P+\nu \nabla^{2} \mathbf{u}+O\left(\mathrm{Ma}^{3}\right),
\end{aligned}
$$

where $P=c_{\mathrm{s}}^{2} \rho / \rho_{0}$ is the pressure, and $\nu=\mu / \rho_{0}$ the kinematic viscosity. Steady solutions of (19) approximate steady solutions of the incompressible Navier-Stokes equations with $O\left(\mathrm{Ma}^{3}\right)$ error, one order in Mach number better than the usual isothermal lattice Boltzmann equation [20]. However, for unsteady flows the compressibility error remains $O\left(\mathrm{Ma}^{2}\right)$, because the difference between the two sets of equilibria in (13) and (18) is only $O\left(\mathrm{Ma}^{3}\right)$, since $\rho=\rho_{0}+O\left(\mathrm{Ma}^{2}\right)$ and $\mathbf{u}=O(\mathrm{Ma})$. In the numerical experiments reported below, the density variations are sufficiently small that there is very little difference between the two schemes. In fact, the two schemes coincide exactly when linearized around a spatially uniform rest state as in Sections 6 and 7.

\section{MULTIPLE RELAXATION TIMES}

The collision matrix $\Omega_{i j}$ appearing in (5) must satisfy many constraints in order to reproduce the isotropic Navier-Stokes equations $[4,24,33,16]$. The easiest way to specify $\Omega_{i j}$ is to transform from the $f_{i}$ to an alternative set of variables, including the hydrodynamic variables $\rho, \mathbf{u}$, and $\Pi$, that should be eigenvectors of the collision matrix. Using the same variables as the author's earlier paper [12], we write

$$
f_{i}=w_{i}\left(\rho+\frac{1}{\theta}(\rho \mathbf{u}) \cdot \boldsymbol{\xi}_{i}+\frac{1}{2 \theta^{2}}(\mathbf{\Pi}-\theta \rho \mathbf{l}):\left(\boldsymbol{\xi}_{i} \boldsymbol{\xi}_{i}-\theta \mathbf{l}\right)\right)+w_{i} g_{i}\left(\frac{1}{4} \mathcal{N}+\frac{3}{8} \boldsymbol{\xi}_{i} \cdot \mathcal{J}\right),
$$

where $\theta=1 / 3$ in lattice units, and $g_{i}=(1,-2,-2,-2,-2,4,4,4,4)^{\top}$. The two "ghost variables" $\mathcal{N}$ and $\mathcal{J}$ are given by the moments

$$
\mathcal{N}=\sum_{i=0}^{8} g_{i} f_{i}, \quad \mathcal{J}=\sum_{i=0}^{8} g_{i} \boldsymbol{\xi}_{i} f_{i},
$$

by analogy with (4). The nine variables $f_{i}$ are thus decomposed into two scalars $\rho$ and $\mathcal{N}$, two vectors $\mathbf{u}$ and $\mathcal{J}$, and a symmetric second rank tensor $\boldsymbol{\Pi}$. Moreover, the lattice vectors appearing in (20), 1, $\boldsymbol{\xi}_{i}, \boldsymbol{\xi}_{i} \boldsymbol{\xi}_{i}-\theta \mathbf{l}, g_{i}$, and $g_{i} \boldsymbol{\xi}_{i}$, are all orthogonal with respect to the weighted inner product with weights $w_{i}$ [12]. The three ghost vectors $g_{i}$ and $g_{i} \boldsymbol{\xi}_{i}$ thus extend the first three tensor Hermite polynomials, $1, \boldsymbol{\xi}_{i}$, and $\boldsymbol{\xi}_{i} \boldsymbol{\xi}_{i}-\theta$ l, to an orthogonal basis for $\mathbb{R}^{9}$. The use of tensor Hermite polynomials is motivated by the work of He and Luo [21] who derived the equilibria in (13), and the weights in (14), for the common isothermal lattice Boltzmann equation from the continuum Boltzmann equation via a truncated expansion in tensor Hermite polynomials.

However, other choices are possible. Benzi et al. [3, 4] used a different set of weights, in which the rest particles associated with $\boldsymbol{\xi}_{0}$ had the same weight as the particles associated with the non-diagonal velocities $\boldsymbol{\xi}_{1,2,3,4}$. Our $\mathcal{N}$ and $\mathcal{J}$ are analogous to the variables $\mu$ and $\boldsymbol{\eta}$ used by Benzi et al. [3, 4]. Lallemand and Luo [25] used yet another set of variables, as introduced by d'Humières [13], based on lattice vectors that are orthogonal with respect to the unweighted $\ell_{2}$ inner product. The discrete equilibria happen to have the elegant representation (20) in terms of tensor Hermite polynomials for the isothermal equation of state with $\theta=1 / 3$ in lattice units; but for general equations of state [12], and especially for varying temperatures, the discrete equilibria do not coincide with truncated expansions in tensor Hermite polynomials. 
In terms of the variables in (20), the lattice Boltzmann equation (5) is equivalent to the coupled system

$$
\begin{aligned}
\partial_{t} \rho+\nabla \cdot(\rho \mathbf{u}) & =0 \\
\partial_{t}(\rho \mathbf{u})+\nabla \cdot \boldsymbol{\Pi} & =0 \\
\partial_{t} \boldsymbol{\Pi}+\nabla \cdot\left(\sum_{i=0}^{8} \boldsymbol{\xi}_{i} \boldsymbol{\xi}_{i} \boldsymbol{\xi}_{i} f_{i}\right) & =-\frac{1}{\tau_{\Pi}}\left(\boldsymbol{\Pi}-\boldsymbol{\Pi}^{(0)}\right), \\
\partial_{t} \mathcal{N}+\nabla \cdot \mathcal{J} & =-\frac{1}{\tau_{N}}\left(\mathcal{N}-\mathcal{N}^{(0)}\right), \\
\partial_{t} \mathcal{J}+\nabla \cdot\left(\sum_{i=0}^{8} g_{i} \boldsymbol{\xi}_{i} \boldsymbol{\xi}_{i} f_{i}\right) & =-\frac{1}{\tau_{J}}\left(\mathcal{J}-\mathcal{J}^{(0)}\right) .
\end{aligned}
$$

No relaxation times appear in $(22 \mathrm{a})$ and $(22 \mathrm{~b})$ because mass and momentum conservation imply that $\rho^{(0)}=\rho$ and $\mathbf{u}^{(0)}=$ $\mathbf{u}$, so the first two right hand sides always vanish. The remaining three relaxation times $\tau_{\Pi}, \tau_{N}$, and $\tau_{J}$ may be adjusted independently.

The hydrodynamic variables $\rho, \mathbf{u}, \boldsymbol{\Pi}$ are coupled to the ghost variables $\mathcal{N}$ and $\mathcal{J}$ by the two terms expressed as sums in (22c) and (22e). The combinations such as $g_{i} \boldsymbol{\xi}_{i} \boldsymbol{\xi}_{i}$ may be expressed in terms of the nine basis vectors as [12]

$$
\begin{aligned}
g_{i} \xi_{i_{x}} \xi_{i_{x}} & =2\left(\xi_{i y} \xi_{i y}-\theta 1_{i}\right)+\frac{2}{3} g_{i}, & g_{i} \xi_{i_{x}} \xi_{i y} & =4 \xi_{i_{x}} \xi_{i y} \\
\xi_{i_{x}} \xi_{i_{x}} \xi_{i_{x}} & =\xi_{i x}, & \xi_{i_{x}} \xi_{i_{x}} \xi_{i_{y}} & =\frac{1}{3} \xi_{i y}+\frac{1}{6} g_{i} \xi_{i_{y}}
\end{aligned}
$$

and their permutations in $x$ and $y$. In particular $\mathcal{J}$ appears in the nonequilibrium stress via (23b) and (22c), for instance

$$
\sum_{i=0}^{8} \xi_{i_{x}} \xi_{i_{x}} \xi_{i y} f_{i}=\sum_{i=0}^{8}\left(\frac{1}{3} \xi_{i y}+\frac{1}{6} g_{i} \xi_{i y}\right) f_{i}=\frac{1}{3} u_{y}+\frac{1}{6} J_{y}
$$

The complete closed system of equations for $\rho, \mathbf{u}, \Pi, \mathcal{N}$, and $\mathcal{J}$ may be found in Ref. [12].

Equation (22c) for the symmetric stress tensor $\Pi$ may be further decomposed into separate equations for the trace (which is a scalar) and the remaining traceless part. The relaxation time $\tau_{\mathrm{b}}$ for the trace $\Pi_{x x}+\Pi_{y y}$ of $\Pi$ determines a bulk viscosity that may be different from the shear viscosity determined by the relaxation time $\tau_{\mathrm{s}}$ for the traceless part of $\Pi$ [25, 14, 11]. This decomposition of the nine $f_{i}$ into six quantities: three scalars, two vectors, and a symmetric traceless rank 2 tensor is now irreducible, meaning that no further decomposition would remain invariant under rotation of the coordinates. Thus $\Omega_{i j}$, as determined by transforming equations (22a-e) back to the $f_{i}$ variables, is determined uniquely by the four free parameters $\tau_{\mathrm{s}}, \tau_{\mathrm{b}}, \tau_{N}$, and $\tau_{J}$.

\section{NUMERICAL IMPLEMENTATION}

The discrete Boltzmann equation (5) is usually implemented computationally as the fully discrete system, or lattice Boltzmann equation

$$
\bar{f}_{i}\left(\mathbf{x}+\boldsymbol{\xi}_{i} \Delta t, t+\Delta t\right)-\bar{f}_{i}(\mathbf{x}, t)=-\left[\Delta t \Omega\left(1+\frac{1}{2} \Delta t \Omega\right)^{-1}\right]_{i j}\left(\bar{f}_{j}(\mathbf{x}, t)-f_{j}^{(0)}(\mathbf{x}, t)\right)
$$

where the $\bar{f}_{i}$ are defined by

$$
\bar{f}_{i}(\mathbf{x}, t)=f_{i}(\mathbf{x}, t)+\frac{1}{2} \Delta t \Omega_{i j}\left(f_{j}(\mathbf{x}, t)-f_{j}^{(0)}(\mathbf{x}, t)\right) .
$$

The term in square brackets in (25) should be interpreted as the components $C_{i j}$ of the fully discrete collision matrix $\mathrm{C}=$ $\Delta t \Omega\left(1+\frac{1}{2} \Delta t \Omega\right)^{-1}$. In the BGK approximation with $\Omega_{i j}=\tau^{-1} \delta_{i j},(25)$ reduces to

$$
\bar{f}_{i}\left(\mathbf{x}+\boldsymbol{\xi}_{i} \Delta t, t+\Delta t\right)-\bar{f}_{i}(\mathbf{x}, t)=-\frac{\Delta t}{\tau+\Delta t / 2}\left(\bar{f}_{i}(\mathbf{x}, t)-f_{i}^{(0)}(\mathbf{x}, t)\right)
$$

which coincides with (2) above. The transformation from $f_{i}$ to $\bar{f}_{i}$ in (26) then coincides with that introduced by He et al. $[22,19]$. 
Equation (25) and its BGK form (27) may be derived by integrating the discrete Boltzmann equation (5) along a characteristic for time $\Delta t$. Approximating the integral of the collison term on the right hand side using the trapezium rule gives

$f_{i}\left(\mathbf{x}+\boldsymbol{\xi}_{i} \Delta t, t+\Delta t\right)-f_{i}(\mathbf{x}, t)=\frac{1}{2} \Delta t \Omega_{i j}\left[f_{j}\left(\mathbf{x}+\boldsymbol{\xi}_{i} \Delta t, t+\Delta t\right)-f_{j}^{(0)}\left(\mathbf{x}+\boldsymbol{\xi}_{i} \Delta t, t+\Delta t\right)+f_{j}(\mathbf{x}, t)-f_{j}^{(0)}(\mathbf{x}, t)\right]+O\left(\Delta t^{3}\right)$.

This equation is not suitable as it stands for a timestepping scheme because the $f_{i}$ at time $t+\Delta t$ also appear on the right hand side; both explicitly, and implicitly through the dependence of $f_{j}^{(0)}\left(\mathbf{x}+\boldsymbol{\xi}_{i} \Delta t, t+\Delta t\right)$ on the $f_{i}$ via $\rho$ and $\mathbf{u}$. However, equation (28) is algebraically identical to the fully explicit equation (25) under the change of variables defined by (26). The solvability conditions (10) imply that the substitution (26) leaves the density and momentum unchanged,

$$
\rho=\sum_{i=0}^{n} \bar{f}_{i}, \quad \rho \mathbf{u}=\sum_{i=0}^{n} \boldsymbol{\xi}_{i} \bar{f}_{i}
$$

so the $f_{i}^{(0)}$ may be computed directly from the $\bar{f}_{i}$, making the $f_{i}$ redundant. In other words, the $\bar{f}_{i}$ at time $t+\Delta t$ are given explicitly in terms of quantities known at time $t$ by equation (25), while the $f_{i}$ at time $t+\Delta t$ would have to be found by solving the system (28) of algebraic equations [22, 19].

In the general case, the right hand side of (25) would be evaluated by projecting onto the lattice vectors associated with $\rho$, $\rho \mathbf{u}, \boldsymbol{\Pi}, \mathcal{N}$, and $\mathcal{J}$ as given in the last section. These lattice eigenvectors define a basis in which $\Delta t \Omega$ and $\left(1+\frac{1}{2} \Delta t \Omega\right)^{-1}$ are both diagonal, so the matrix $C_{i j}$ in (25) becomes simply $\Delta t /\left(\tau_{\lambda}+\frac{1}{2} \Delta t\right)$ multiplying each eigenvector $\lambda$ as in (27). For instance, a collision operator that changes only the relaxation time $\tau_{N}$ for the ghost variable $\mathcal{N}$ may be implemented as

$$
\begin{aligned}
\bar{f}_{i}\left(\mathbf{x}+\boldsymbol{\xi}_{i} \Delta t, t\right. & +\Delta t)-\bar{f}_{i}(\mathbf{x}, t)=-\frac{\Delta t}{\tau+\Delta t / 2}\left(\bar{f}_{i}(\mathbf{x}, t)-f_{i}^{(0)}(\mathbf{x}, t)\right) \\
& -\left(\frac{\Delta t}{\tau_{N}+\Delta t / 2}-\frac{\Delta t}{\tau+\Delta t / 2}\right) w_{i} g_{i} \frac{1}{4} \sum_{j=0}^{8} g_{j}\left(\bar{f}_{j}(\mathbf{x}, t)-f_{j}^{(0)}(\mathbf{x}, t)\right) .
\end{aligned}
$$

Since $\mathcal{N}^{(0)}=\sum_{j=0}^{8} g_{j} f_{j}^{(0)}(\mathbf{x}, t)=0$ for the equilibria given above, it is only necessary to compute $\mathcal{N}=\sum_{j=0}^{8} g_{j} \bar{f}_{j}(\mathbf{x}, t)$. This would typically be implemented in the same loop that computes $\rho$ and $\mathbf{u}$ from the $\bar{f}_{j}$ in order to evaluate the equilibria $f_{i}^{(0)}$.

\subsection{Reducing round-off error}

For small Mach numbers the density is almost uniform, $\rho=\rho_{0}+O\left(\mathrm{Ma}^{2}\right)$, and the macroscopic fluid velocity is small, $\mathbf{u}=O(\mathrm{Ma})$. The two sets of distribution functions appearing in (25) are therefore both almost equal to the rest state equilibria $\rho_{0} w_{i}$. In other words, $\bar{f}_{i}=\rho_{0} w_{i}+O(\mathrm{Ma})$ and $f_{i}^{(0)}=\rho_{0} w_{i}+O(\mathrm{Ma})$, so the difference $\bar{f}_{i}-f_{i}^{(0)}$ is only $O(\mathrm{Ma})$. The loss of numerical precision arising from computing the difference between two nearly equal quantities may be reduced by analytically subtracting out the $\rho_{0} w_{i}$ contribution to $f_{i}^{(0)}$ and $\bar{f}_{i}$, and evolving only the difference $\bar{f}_{i}-\rho_{0} w_{i}$, as proposed by Skordos [30]. The macroscopic variables $\rho$ and $\mathbf{u}$ may be reconstructed as

$$
\rho=\rho_{0}+\sum_{i=0}^{n}\left(\bar{f}_{i}-\rho_{0} w_{i}\right), \quad \rho \mathbf{u}=\sum_{i=0}^{n} \boldsymbol{\xi}_{i}\left(\bar{f}_{i}-\rho_{0} w_{i}\right)
$$

Without this rearrangement, the convergence of the $256^{2}$ simulations shown in Fig. 4 was visibly affected by numerical rounding error in IEEE 64 bit (16 digit) floating point arithmetic. The results presented below were verified by comparisons between solutions obtained using different platforms and compilers, and with a few solutions computed using IEEE 128 bit arithmetic.

\section{DOUBLY-PERIODIC SHEAR LAYERS}

Minion and Brown [28] studied the performance of various numerical schemes in under-resolved simulations of the 2D incompressible Navier-Stokes equations. Their initial conditions corresponded to a pair of perturbed shear layers,

$$
\begin{aligned}
& u_{x}= \begin{cases}\tanh (\kappa(y-1 / 4)), & y \leq 1 / 2, \\
\tanh (\kappa(3 / 4-y)), & y>1 / 2\end{cases} \\
& u_{y}=\delta \sin (2 \pi(x+1 / 4)),
\end{aligned}
$$



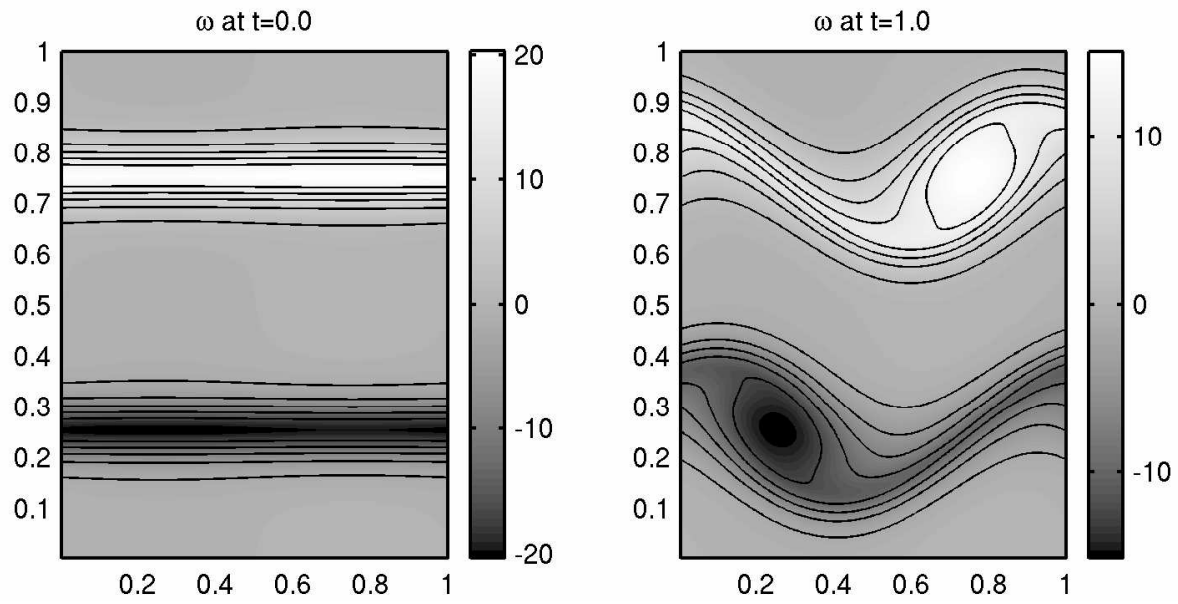

FIG. 3. Vorticity during the roll up of a perturbed doubly-periodic shear layer. The left figure shows the initial conditions from (32), while the right figure shows the rolled up shear layers at $t=1$. This solution was computed with the BGK collision operator for Mach number Ma $=\sqrt{3} / 2000$ and Reynolds number $\operatorname{Re}=1000$ on a $256^{2}$ lattice.

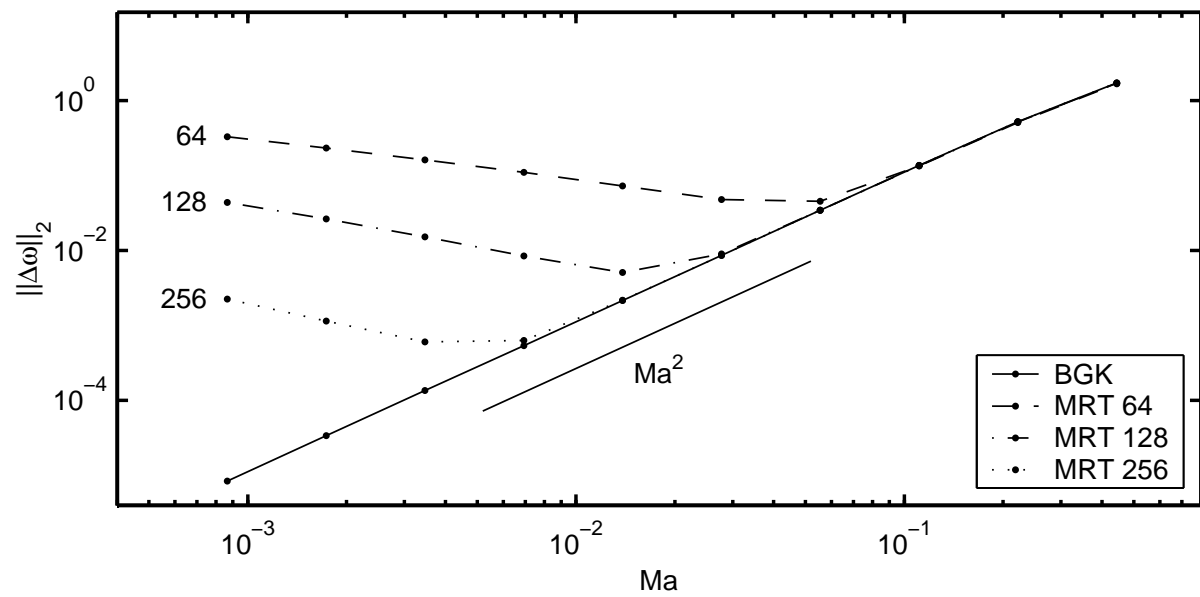

FIG. 4. Divergence of vorticity at $t=1.0$ as $\mathrm{Ma} \rightarrow 0$ with the multiple relaxation time (MRT) collision operator. Results are shown for Re $=1000$ on $64^{2}, 128^{2}$, and $256^{2}$ lattices. The difference $\Delta \omega$ is between the solution for given Mach number and the incompressible limit obtained from the BGK solution on each lattice by Richardson extrapolation. The compressibility error for the BGK solution error decays as $O\left(\mathrm{Ma}^{2}\right)$, with no visible difference between the three lattices. For the MRT collision operator, the error begins to increase again for sufficiently small Mach numbers. For high resolutions the error diverges approximately as $O\left(\mathrm{Ma}^{-1}\right)$.

in the doubly periodic domain $0 \leq x, y \leq 1$. The parameter $\kappa$ controls the width of the shear layers, and $\delta$ the magnitude of the initial perturbation. The shear layers roll up due to a Kelvin-Helmholtz instability excited by the $O(\delta)$ perturbation in $u_{y}$. The simulations presented below used $\kappa=20, \delta=0.05$, and Reynolds numbers of 1000 and 5000. Typical plots of the vorticity $\omega=\partial_{x} u_{y}-\partial_{y} u_{x}$ for $t=0.0$ and $t=1.0$ are shown in Fig. 3. All the vorticity fields shown in this paper were computed from the velocities $u_{x}$ and $u_{y}$ at lattice points by spectrally accurate differentiation using the fast Fourier transform library FFTW [15]. These comparatively thick shear layers show no sign of forming the spurious vortices found by Minion and Brown [28] with $\kappa=80$ on a $128^{2}$ grid at $\mathrm{Re}=10000$.

Figure 4 shows the $\ell_{2}$ norm of the error in the vorticity, $\|\Delta \omega\|_{2}$, due to a finite Mach number for various Mach numbers down to $\sqrt{3} / 2000 \approx 8.6 \times 10^{-4}$. The comparison solution for the incompressible $(\mathrm{Ma} \rightarrow 0)$ limit was obtained by Richardson extrapolation from solutions with $\mathrm{Ma}=\sqrt{3} / 2000$ and $\mathrm{Ma}=\sqrt{3} / 4000$ using the BGK collision operator, assuming an $O\left(\mathrm{Ma}^{2}\right)$ dependence of the error. This assumption is verified by the $\mathrm{Ma}^{2}$ slope of the compressibility error shown in Fig. 4 . Moreover, the contour plots of $\Delta \omega$ in Fig. 5 show an essentially identical spatial pattern in the compressibility error at four different Mach numbers. The compressibility error is largest where the streamlines are curved, so the centrifugal force must be balanced by a pressure gradient, and vanishes in the middle of the shear layers where the streamlines are nearly straight.

The dotted lines in Fig. 4 show the results of computations with a multiple relaxation time (MRT) collision operator. The relaxation time for the scalar ghost variable $\mathcal{N}$ was chosen to be $\tau_{N}=\frac{1}{2} \Delta t$, while all other relaxation times were equal and determined by the viscosity. This value for $\tau_{N}$ gives the most rapid decay of $\mathcal{N}$ towards zero in a spatially uniform state, as 

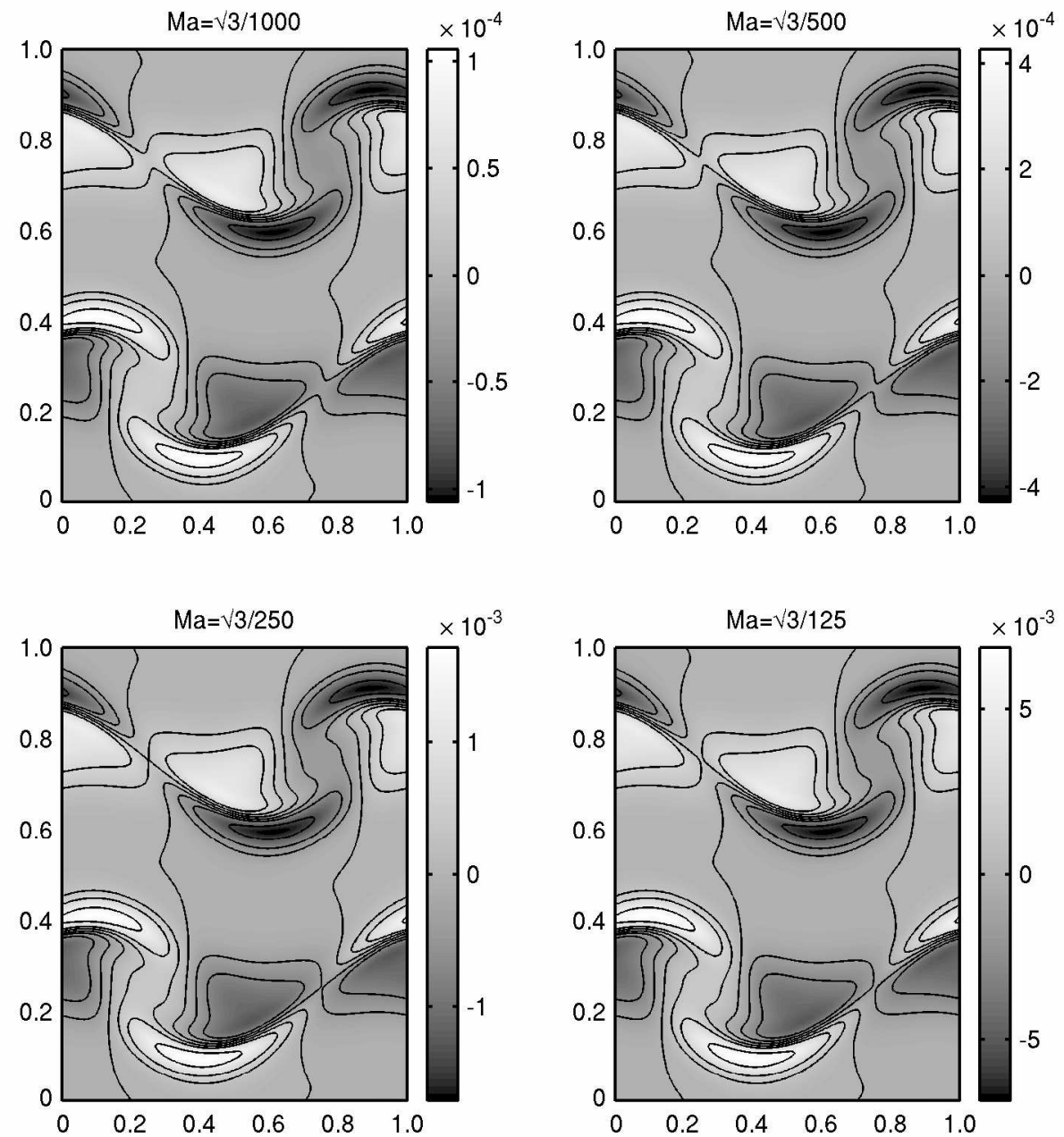

FIG. 5. Convergence of vorticity at $t=1.0$ as $\mathrm{Ma} \rightarrow 0$ with the BGK collision operator. The difference $\Delta \omega$ between the solution for a given Mach number and the incompressible limit, as obtained by Richardson extrapolation from solutions at the two Mach numbers $\sqrt{3} / 2000$ and $\sqrt{3} / 4000$, decays like $O\left(\mathrm{Ma}^{2}\right)$ as $\mathrm{Ma} \rightarrow 0$. All computations were performed with $\mathrm{Re}=1000$ on a $256^{2}$ lattice. Notice that the spatial patterns are very similar in all four plots, although the amplitudes decrease proportionally to $\mathrm{Ma}^{2}$ as shown by the labels on the color bars. 

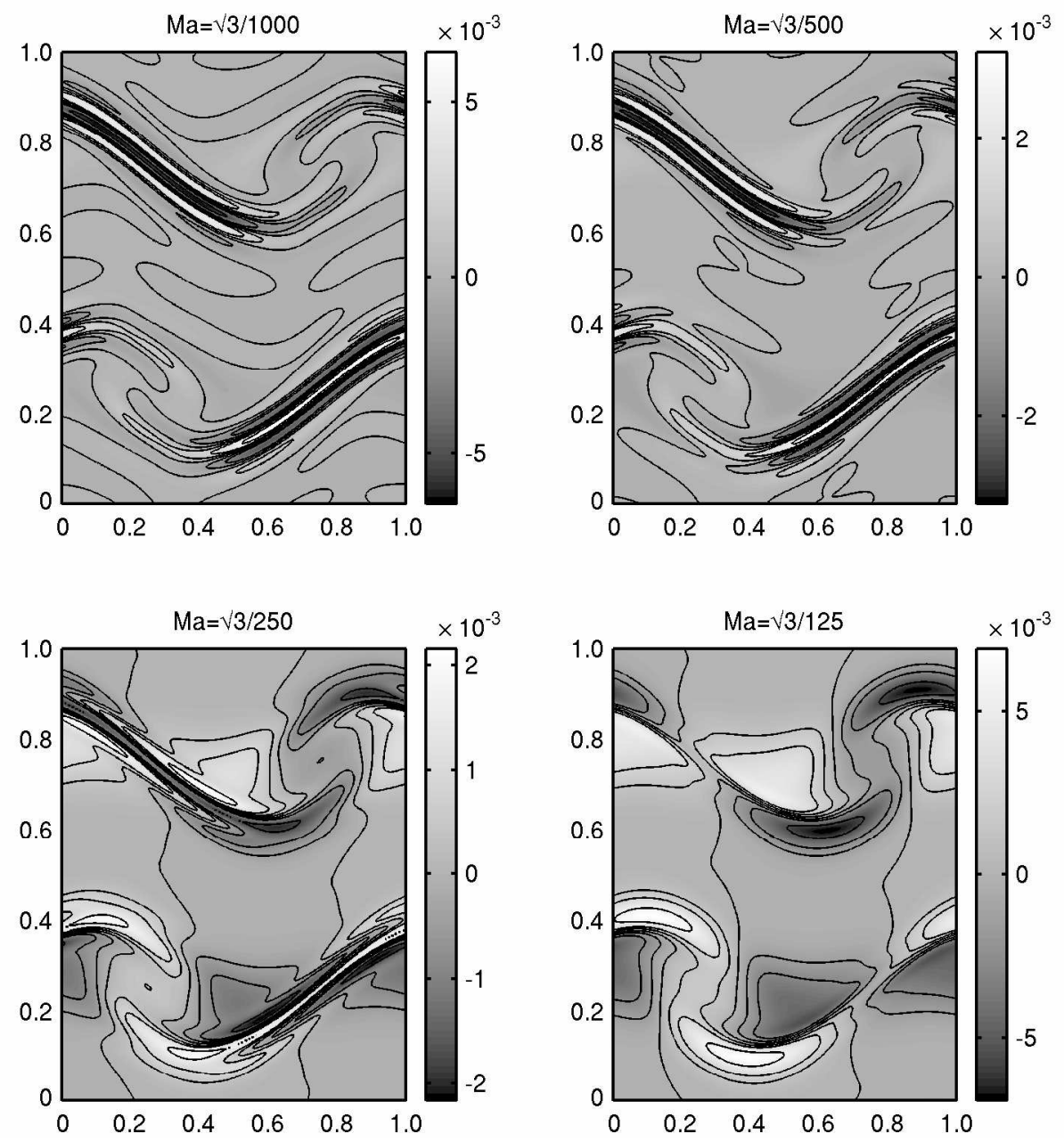

FIG. 6. Divergence of vorticity at $t=1.0$ as $\mathrm{Ma} \rightarrow 0$ with the multiple relaxation time (MRT) collision operator. The difference $\Delta \omega$ between the solution for a given Mach number and the incompressible limit, as obtained by Richardson extrapolation from solutions at the two Mach numbers $\sqrt{3} / 2000$ and $\sqrt{3} / 4000$, does not converge as $\mathrm{Ma} \rightarrow 0$. All computations were performed with $\mathrm{Re}=1000$ on a $256^{2}$ lattice. For the largest Mach number, $\Delta \omega$ resembles the spatial patterns in Fig. 5. For smaller Mach numbers (upper panels) the pattern is distinctly different, with $\Delta \omega$ largest around the shear layers, and the amplitude begins to increase again, as shown by the labels on the color bars.

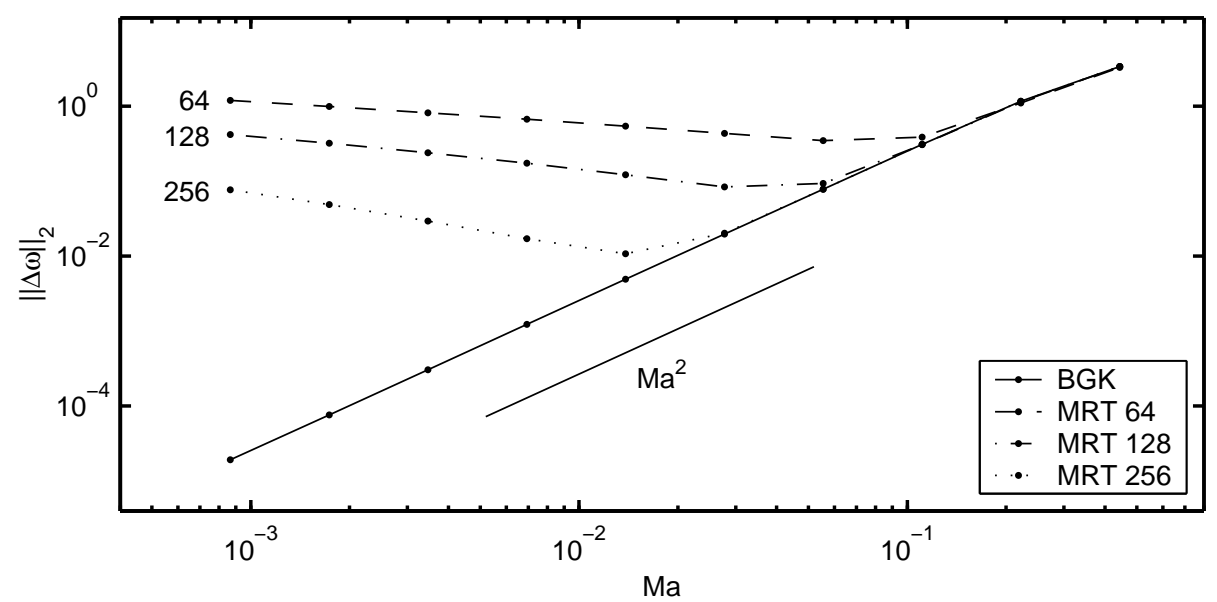

FIG. 7. Divergence of vorticity at $t=1.0$ as $\mathrm{Ma} \rightarrow 0$ with the multiple relaxation time (MRT) collision operator. Results are shown for Re $=5000$ on $64^{2}, 128^{2}$, and $256^{2}$ lattices. The errors are systematically larger than in Fig. 4, for Re $=1000$, which is plotted with the same axes. As before, the difference $\Delta \omega$ is between the solution for given Mach number and the incompressible limit obtained from the BGK solution on each lattice by Richardson extrapolation. The compressibility error for the BGK solution error decays as $O\left(\mathrm{Ma}^{2}\right)$, with no visible difference between the three lattices. For the MRT collision operator, the error begins to increase again for sufficiently small Mach numbers. For high resolutions the error diverges approximately as $O\left(\mathrm{Ma}^{-1}\right)$. 

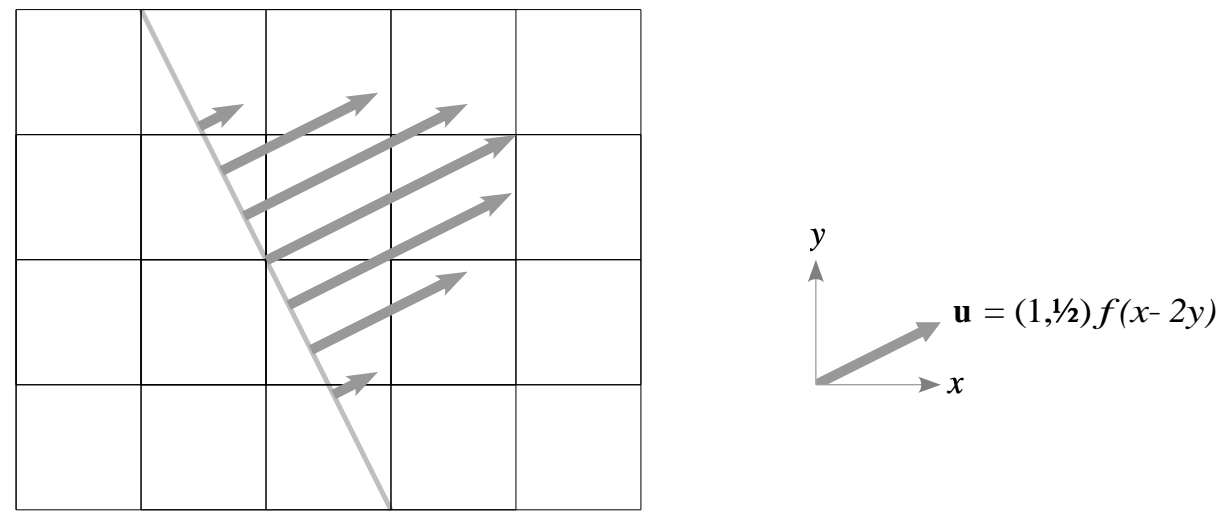

FIG. 8. Sketch of the inclined jet, the form of solution considered in (33), corresponding to unidirectional Poiseuille-type flow inclined at an angle of $\tan ^{-1}(1 / 2) \approx 27^{\circ}$ to the horizontal axis.

recommended by Higuera et al. [24] and Succi [32]. Lallemand and Luo [25] took $\tau_{N}$ slightly smaller than $\frac{1}{2} \Delta t$, so the decay rate $\gamma_{N}=\Delta t /\left(\tau_{N}+\Delta t / 2\right)$ is slightly greater than one.

For this MRT collision operator, the vorticity on a fixed lattice at a fixed Reynolds number no longer converges to an incompressible limit as the Mach number tends to zero. Instead, the error begins to increase again for sufficiently small Mach numbers. The growth rate is approximately $O\left(\mathrm{Ma}^{-1}\right)$, with the actual exponent tending closer to -1 on finer lattices. Figure 6 shows the error in vorticity at various Mach numbers with the above MRT collision operator. For the largest Mach number shown, $\mathrm{Ma}=\sqrt{3} / 125 \approx 0.014$, the error looks very similar to the compressibility error with the BGK approximation as shown in Fig. 5. In particular, the compressibility error in the vorticity is concentrated where the streamlines are curved, and vanishes where they are straight. However, for smaller Mach numbers the error in the vorticity with the MRT collision operator looks noticeably different. It is concentrated around the shear layers, where the BGK compressibility error was small, and is small near the two vortices where the BGK compressibility error was largest. The same behavior occurs with $\mathrm{Re}=5000$, although the errors are systematically larger, as shown in Fig. 7.

For the smallest Mach number shown, $\mathrm{Ma}=\sqrt{3} / 1000 \approx 0.0017$, the greatest deviation of the density from its mean value $\rho_{0}=1$ was $2 \times 10^{-6}$, and the maximum density deviation between the BGK and MRT computations was even smaller, less than $2 \times 10^{-11}$. It is therefore not surprising that the results of computations using the pseudo-incompressible equilibria from (18) were visually indistinguishable from those using the more common isothermal equilibria from (13). In particular, the pseudo-incompressible computations suffered from the same divergence of the vorticity in the limit $\mathrm{Ma} \rightarrow 0$.

\section{LINEAR THEORY FOR AN INCLINED JET}

The previous computations become expensive in the small Mach number limit, because $O\left(\mathrm{Ma}^{-1}\right)$ timesteps are required to reach a fixed multiple of an eddy turnover time. Fortunately, the observed divergence at small Mach number also occurs in a linearized system, for which each timestep is equivalent to a multiplication by a time-invariant matrix. Thus the computation is equivalent to computing the $O\left(\mathrm{Ma}^{-1}\right)$ power of a matrix, which may be achieved in $O\left(\log \mathrm{Ma}^{-1}\right)$ operations by successive squaring [17], or in principle in $O(1)$ operations by diagonalising the matrix.

Moreover, the discrepancies visible in Fig. 6 around the almost straight shear layers suggest that a unidirectional flow resembling Poiseuille flow may be sufficient. No unexpected behavior occurs unidirectional flows aligned with the lattice, or aligned at $45^{\circ}$ to the lattice. This is consistent with the discrepancies plotted in Fig. 6, in which the error appears to vanish where the shear layer is nearly horizontal. However, unidirectional flows inclined at other angles to the lattice, for instance with slope $1 / 2$, do show unexpected behavior. This dependence on inclination is due to the anisotropic coupling between the ghost and hydrodynamic variables through (22c) and (22e).

We therefore seek solutions of the form

$$
f_{i}(\mathbf{x}, t)=f_{i}^{(0)}+h_{i}(x-2 y, t)
$$

where $f_{i}^{(0)}$ denotes a uniform rest state. For suitable values of the $h_{i}$ these solutions describe a small amplitude unidirectional jet, with a velocity of the form $\mathbf{u}=\left(\hat{\mathbf{x}}+\frac{1}{2} \hat{\mathbf{y}}\right) u(x-2 y)$, inclined at an angle of $\tan ^{-1}(1 / 2) \approx 27^{\circ}$ to the horizontal axis as sketched in Fig. 8. Here $\hat{\mathbf{x}}$ and $\hat{\mathbf{y}}$ are unit vectors in the $x$ and $y$ directions. Incompressibility requires the flow to be invariant in the direction parallel to $\mathbf{u}$, i.e. $\mathbf{u} \cdot \nabla \mathbf{u}=0$ as in Poiseuille flow, so $\mathbf{u}$ and the other variables should be functions of the perpendicular coordinate $\zeta=x-2 y$ only. The $\zeta$ axis is indicated by the pale gray line in Fig. 8 . 


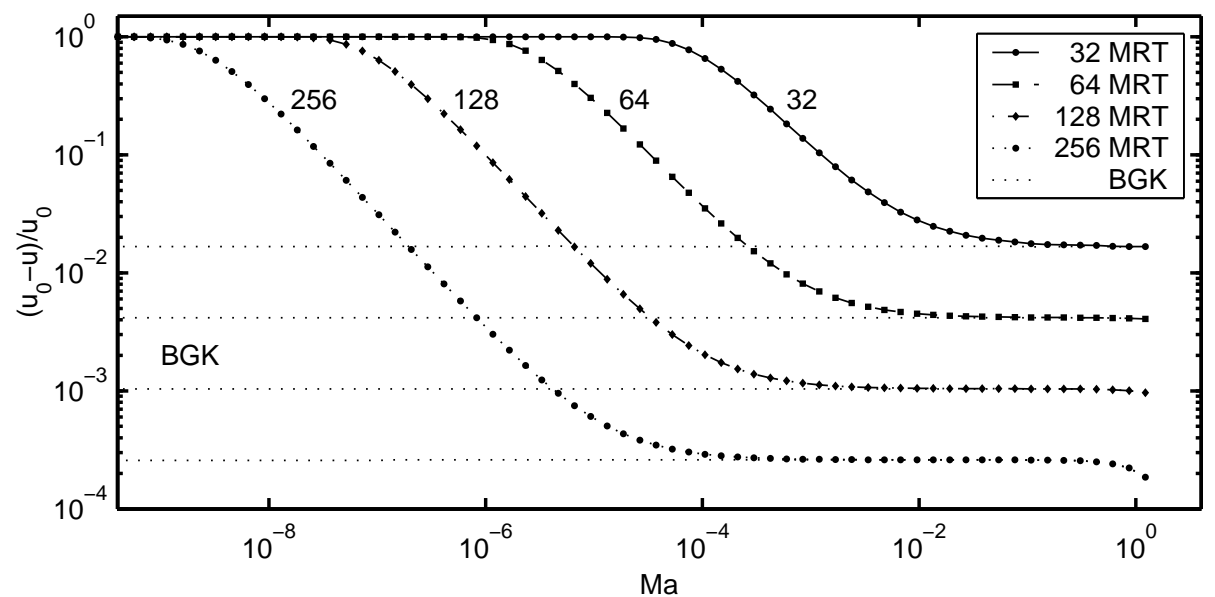

FIG. 9. Excessive decay of peak jet speed $u$ at $t=1.0$ as $\mathrm{Ma} \rightarrow 0$ with the multiple relaxation time (MRT) collision operator. Results are shown for $\operatorname{Re}=2000$ on lattices with 32,64, 128, and 256 points. For moderate Mach numbers the error is $O\left(N^{2}\right)$, consistent with second order spatial accuracy. For sufficiently small Mach numbers the relative error $\left(u_{0}-u\right) / u_{0}$ begins to diverge as $O\left(\mathrm{Ma}^{-1}\right)$, until it reaches a maximum of one when the jet speed has decayed to zero. By contrast, the errors in the BGK solutions (dots) all tend to $O\left(N^{2}\right)$ limits as Ma $\rightarrow 0$.

Equation (33) may be further simplified by assuming a sinusoidal spatial variation, so that

$$
f_{i}(\mathbf{x}, t)=f_{i}^{(0)}+h_{i}(t) e^{i k_{x}(x-2 y)},
$$

where the $h_{i}$ are now functions of time only. The coordinates $x$ and $y$ should be restricted to integer multiples of the lattice spacing, $x=I \Delta x$ and $y=J \Delta y$. Substituting into the linearized fully discrete lattice Boltzmann equation (25), we obtain a $9 \times 9$ matrix equation for the $h_{i}$,

$$
h_{i}(t+\Delta t)=M_{i j} h_{j}(t),
$$

where the matrix $\mathrm{M}$ with components $M_{i j}$ implicitly depends on $\tau$, and $\tau$ in turn depends on the Mach number. Suitable initial conditions for an inclined jet are

$$
h_{i}(0)=3 w_{i} \mathbf{u} \cdot \boldsymbol{\xi}_{i}=3 w_{i}\left(\xi_{i x}+\frac{1}{2} \xi_{i y}\right)
$$

with the fluid velocity $\mathbf{u}=\hat{\mathbf{x}}+\frac{1}{2} \hat{\mathbf{y}}$ being perpendicular to the direction of spatial variation, as sketched in Fig. 8 . We take the jet to be of unit wavelength in the $x$ direction, so the $x$ wavenumber $k_{x}=2 \pi$. If the jet is allowed to decay viscously for unit time its velocity should decrease by a factor

$$
\exp \left(-5 k_{x}^{2} / \mathrm{Re}\right)=\exp \left(-20 \pi^{2} / \mathrm{Re}\right) .
$$

The factor of 5 in the exponent is due to the wavenumber in the $y$ direction being $k_{y}=2 k_{x}$, from the spatial dependence in (33), so the modulus of the wave vector is $|\mathbf{k}|=\sqrt{5} k_{x}=2 \pi \sqrt{5}$.

Translating into lattice units, the unit macroscopic wavelength in the $x$ direction may be divided into $N$ lattice intervals of width $\Delta x=N^{-1}$. A particle traveling with unit lattice speed thus takes $N$ timesteps to cross the lattice, and a sound wave takes $\sqrt{3} N$ timesteps since $\theta=c_{\mathrm{s}}^{2}=1 / 3$ in lattice units. Thus a unit macroscopic time interval, defined as the time the fluid takes to travel a unit distance with unit macroscopic speed, corresponds to $\sqrt{3} N /$ Ma timesteps.

Figure 9 shows the fractional error in the jet speed, determined by projecting the $h_{i}$ after $\sqrt{3} N /$ Ma timesteps onto the lattice vector $\xi_{i x}+\frac{1}{2} \xi_{i y}$, relative to the exact formula (37), for various Mach numbers Ma and numbers of lattice points $N$. For larger Mach numbers the error is proportional to $N^{-2}$, consistent with the second order spatial accuracy of the scheme. This scaling is confirmed by replotting the data using the variables $N^{2}\left(u_{0}-u\right) / u_{0}$ and $N^{3}$ Ma in Fig. 10, for which the data collapse onto a single curve. However, for sufficiently small Mach numbers the error begins to diverge in proportion to $\mathrm{Ma}^{-1}$ with the multiple relaxation time (MRT) collision operator. Again, we used $\tau_{N}=1 / 2$ in lattice units, while $\tau_{J}=\tau=\sqrt{3} \mathrm{MaN} / \mathrm{Re}$.

Figures 9 and 10 both show data for the MRT collision operator where only $\tau_{N}=1 / 2$ differs from the BGK collision operator. Changing $\tau_{J}$ to be the same as $\tau_{N}$ caused very little difference. Other values of $\tau_{N}$ and $\tau_{J}$ also give similar results. In fact, the behavior for varying $\tau_{N}$ may also be collapsed onto a single curve by using $N^{3} \mathrm{Ma} / \tau_{N}$ as the independent variable, instead of $N^{3} \mathrm{Ma}$ as in Fig. 10. The factor of $N^{3}$ implies that the error at fixed Mach number is third order in space, consistent with the error being due to Burnett terms in the Chapman-Enskog expansion [7, 34]. 


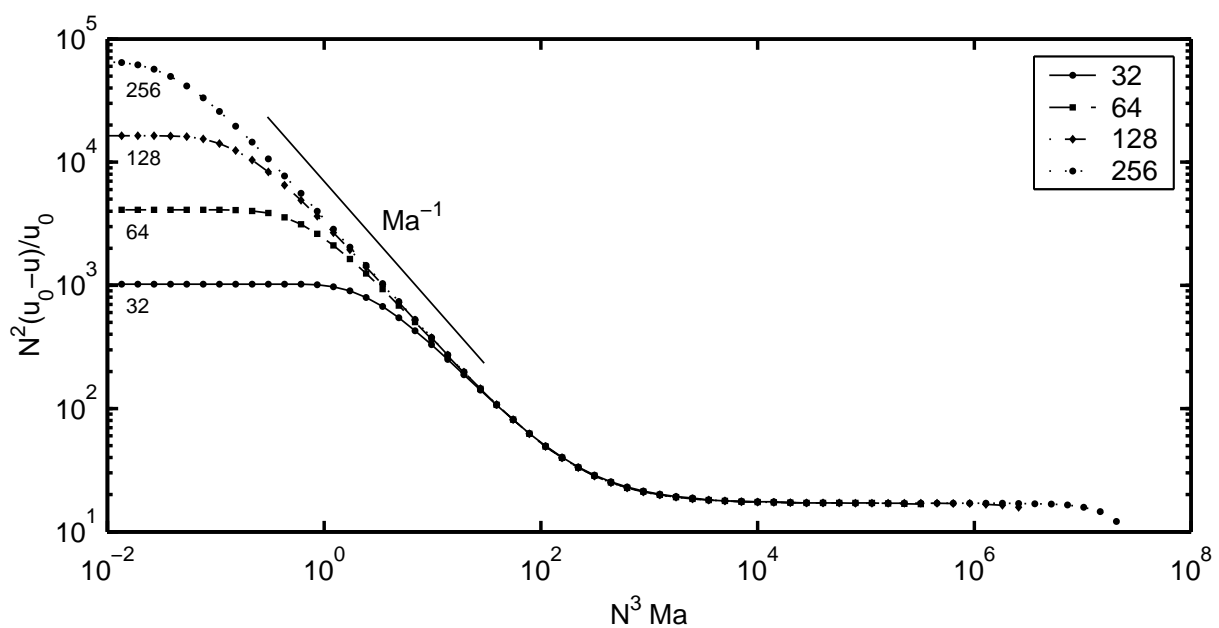

FIG. 10. The above data may be collapsed using the variables $N^{2}\left(u_{0}-u\right) / u_{0}$ and $N^{3}$ Ma. Results are shown for Re $=2000$ on lattices with 32,64 , 128 , and 256 points. The error increases proportional to $\mathrm{Ma}^{-1}$ as indicated by the sloping line.

\section{EIGENVALUE PROBLEM FOR THE INCLINED JET}

The behavior of a fully discrete lattice Boltzmann equation such as (25) is more commonly analysed as an eigenvalue problem [12, 24, 25, 26, 2, 10, 31], instead of as an initial value problem for specific initial conditions as above. Assuming an exponential dependence in time for the $h_{i}$ with growth rate $\sigma$, (35) becomes a $9 \times 9$ matrix eigenvalue problem with eigenvalue $\lambda=e^{\sigma \Delta t}$,

$$
e^{\sigma \Delta t} h_{i}=M_{i j} h_{j}
$$

The constants $h_{i}$ are the eigenvector corresponding to the eigenvalue $\lambda$. This eigenvalue problem is not analytically tractable, involving the roots of a ninth degree polynomial that does not readily factorize, but may be easily solved numerically by the QR iteration algorithm $[17,1]$.

For the BGK collision operator, the eigenvalues $\lambda$ all tend to the unit circle as $\tau \rightarrow 0$ for fixed wavenumber $k$. In other words, $|\lambda|=1-O(\tau)$, which implies $1-|\lambda|=O(\mathrm{Ma})$ at fixed Reynolds number, since $\tau=\sqrt{3} N \mathrm{Ma} / \mathrm{Re}$. The latter relation is plotted as the two straight lines in Fig. 11. Thus the moduli of the eigenvalues of the matrix $\mathrm{M}^{(\sqrt{3} N / \mathrm{Ma})}$, arising from taking $O\left(\mathrm{Ma}^{-1}\right)$ timesteps to cover a unit macroscopic time interval, tend to nonzero values in the $\mathrm{Ma} \rightarrow 0$ limit. This result basically reflects the fact that, for any constant $s$,

$$
(1-s \mathrm{Ma})^{(1 / \mathrm{Ma})} \rightarrow e^{-s} \text { as } \mathrm{Ma} \rightarrow 0,
$$

and the moduli of the eigenvalues of the matrix $\mathrm{M}(\mathrm{Ma})$ are all asymptotically of the form $|\tau| \sim 1-s \mathrm{Ma}+O\left(\mathrm{Ma}^{2}\right)$ as $\mathrm{Ma} \rightarrow 0$.

By contrast, the moduli of all but one of the eigenvalues of the MRT collision operator with fixed $\tau_{N}$ and/or $\tau_{J}$ tend to values strictly less than one in the $\tau \rightarrow 0$ limit, as shown by the dots in Fig. 11. The moduli of the corresponding eigenvalues of the matrix $\mathrm{M}^{(\sqrt{3} N / \mathrm{Ma})}$ therefore all tend to zero in the small Mach number limit. The exception is the one MRT eigenvalue shown approaching the unit circle in Fig. 11. This eigenvalue is given approximately (for $\tau_{N}=1 / 2$ and $\tau_{J}=\tau$ ) by

$$
\lambda=-1+\tau\left(4-100 k_{x}^{2} / 57+O\left(k_{x}^{4}\right)\right)+O\left(\tau^{2}\right),
$$

where $\tau=\sqrt{3} \mathrm{NMa} / \mathrm{Re}$ is the collision time associated with the momentum flux $\Pi$. The corresponding eigenvalue of $\mathrm{M}^{(\sqrt{3} N / \mathrm{Ma})}$ therefore has a modulus given approximately by

$$
|\lambda|=\left[1-\sqrt{3} N \frac{\mathrm{Ma}}{\operatorname{Re}}\left(4-\frac{100}{57} k_{x}^{2}\right)\right]^{(\sqrt{3} N / \mathrm{Ma})} \rightarrow \exp \left(\frac{4 N^{2}\left(25 k^{2}-57\right)}{19 \mathrm{Re}}\right) \text { as } \mathrm{Ma} \rightarrow 0 .
$$

Substituting $k_{x}=2 \pi / N$ into the above,

$$
|\lambda| \rightarrow \exp \left(\frac{-228 N^{2}+400 \pi^{2}}{19 \operatorname{Re}}\right) \text { as } \mathrm{Ma} \rightarrow 0 .
$$




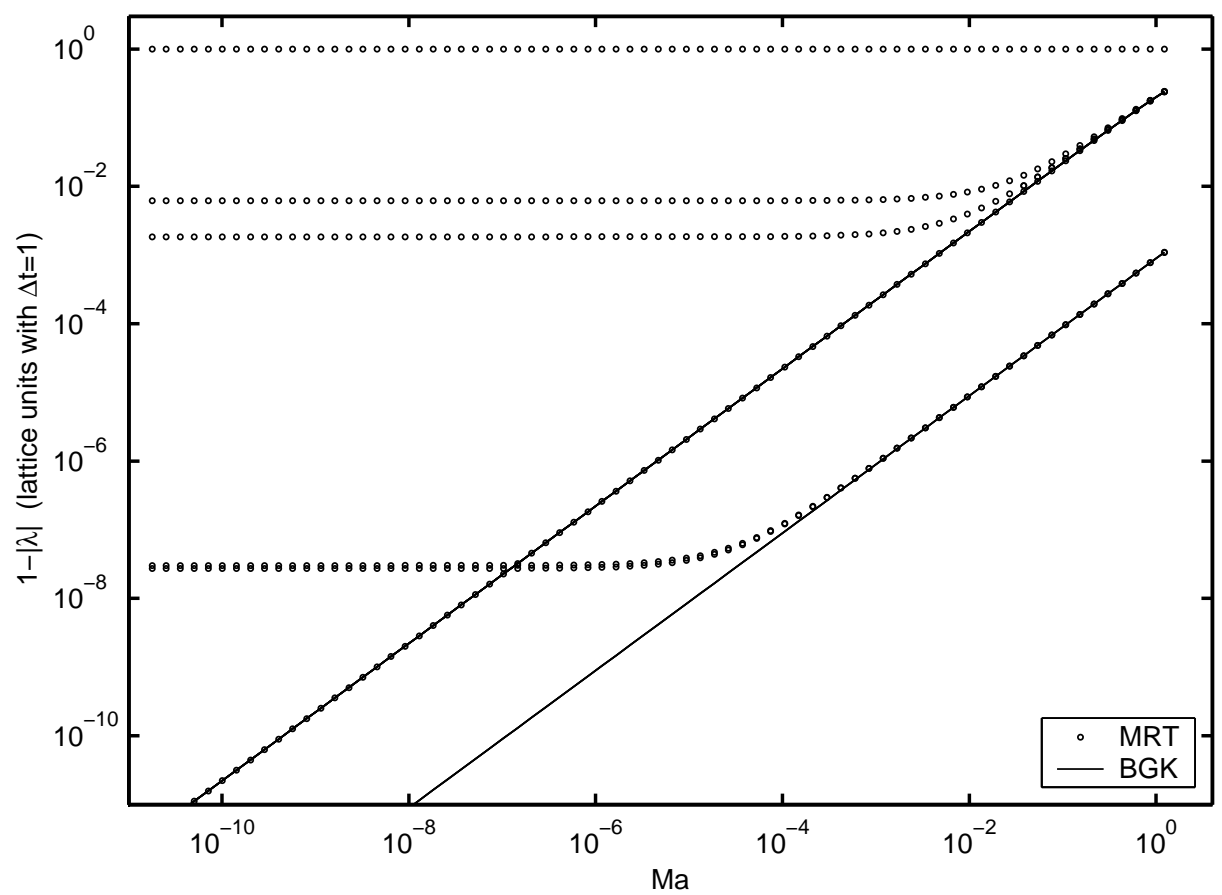

FIG. 11. The eigenvalues of the BGK collision operator (lines) convergence towards the unit circle $(1-|\lambda| \rightarrow 0)$ as Ma $\rightarrow 0$. The three hydrodynamic eigenvalues associated with $\rho$ and $\mathbf{u}$ coincide on the lower line, and the other six eigenvalues coincide on the upper line. All but one of the eigenvalues of the MRT collision operator (plotted for $\tau_{N}=\Delta t / 2$ ) tend to limits with moduli strictly less than one (dots approaching the horizontal). The exception is the single eigenvalue computed in (40) that coincides with one eigenvalue of the BGK collision operator (superimposed diagonal dots and lines).

While this limit is not zero, it does become exponentially small as $N$ is increased. Thus the jet speeds plotted in Fig. 9 do not quite tend to zero, because a finite fraction of the initial conditions project onto the eigenmode associated with the eigenvalue $\lambda$ above that does tend to a finite limit, but they do become exponentially small as the grid is refined (i.e. as $N$ is increased). For $N=64$ and $\operatorname{Re}=2000$ the limiting value of the nonzero eigenvalue as $\mathrm{Ma} \rightarrow 0$ is $\lambda \approx 2.4 \times 10^{-11}$.

Although the matrices $M$ are all non-normal, meaning that $M M^{\top} \neq M^{\top} M$, none of the eigenvalues are significantly illconditioned, in the sense that the cosines of the angles between left and right eigenvectors are never close to zero [17]. Thus no unusual behavior associated with ill-conditioned eigenvalues of non-normal matrices occurs. Moreover, the eigenvalues are all distinct in the complex plane, although their moduli often coincide, as in Fig. 11 where there are three distinct eigenvalues with one modulus (lower diagonal line) for the BGK collision operator, and six distinct eigenvalues with a larger modulus (upper diagonal line).

\section{INTRODUCING GHOST REYNOLDS NUMBERS}

The convergence difficulties described above may be avoided by associating fixed "ghost Reynolds numbers" $R_{N}$ and $R_{J}$ with the non-hydrodynamic modes, and scaling the non-hydrodynamic relaxation times $\tau_{N}=\sqrt{3} \mathrm{NMa} / R_{N}$ and $\tau_{J}=$ $\sqrt{3} N \mathrm{Ma} / R_{J}$ with the Mach number in the same way as the stress relaxation time. Solutions of the resulting lattice Boltzmann equation do then converge to an incompressible limit as $\mathrm{Ma} \rightarrow 0$. Moreover, the compressibility error for a given Mach number appears to be almost independent of $R_{N}$ and $R_{J}$, even when $R_{N}=1$ and $R e=1000$, provided the compressibility error is measured with respect to the incompressible limit computed with the same $\operatorname{Re}, R_{N}$, and $R_{J}$. In other words, the rate of convergence to an incompressible limit is almost independent of the ghost Reynolds numbers, the data collapsing onto single lines in the analogues of figures 4 and 7, but the limiting solutions themselves depend on the ghost Reynolds numbers. These incompressible limiting solutions in turn converge to a universal limit, the true solution for given Mach number and (hydrodynamic) Reynolds number that is independent of $R_{N}$ and $R_{J}$, as the lattice is refined. This convergence is fourth order in space, as illustrated in Fig. 12. The spatial convergence rate is one order higher than that shown in Fig. 10 for solutions with the unmodified multiple relaxation time collision operators at fixed Mach number, due to the extra factor of $N$ multiplying the collision time in the formula $\tau_{N}=\sqrt{3} N \mathrm{Ma} / R_{N}$. Returning to the numerical experiments illustrated in Fig. 1, similar gains in stability to those achieved by setting $\tau_{\mathrm{g}}=\Delta t / 2$ may be obtained by setting $R_{N}=100$, while $R_{J}=\operatorname{Re}=30000$. 


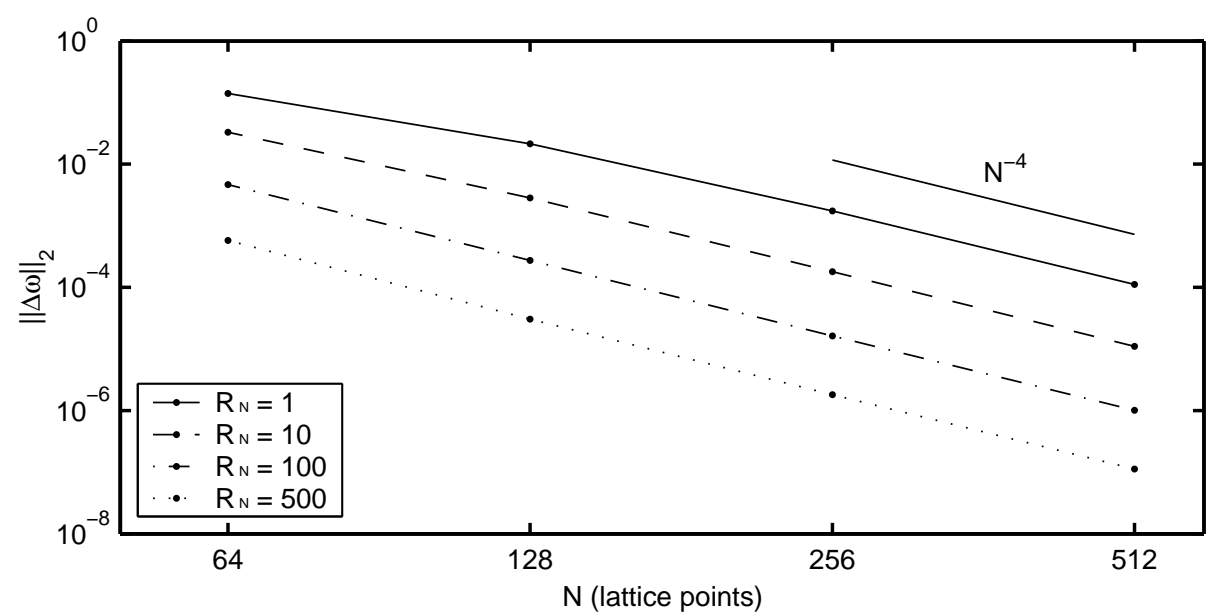

FIG. 12. Vortex rollup solutions computed with Mach number $\mathrm{Ma}=\sqrt{3} / 1000$, hydrodynamic Reynolds number Re $=1000$, and ghost Reynolds number $R_{N}=1,10,100,500$ all converge with fourth order accuracy towards the BGK solution $\left(R_{N}=\mathrm{Re}=1000\right)$ under refinement of the lattice. The other ghost Reynolds number was held at $R_{J}=1000$.

\section{CONCLUSION}

Most lattice Boltzmann equations contain more variables than are necessary to recover the Navier-Stokes equations. These additional degrees of freedom appear as non-hydrodynamic or "ghost" variables. The common BGK collision operator may be generalised so that relaxation times for the ghost variables may be adjusted independently of the stress relaxation time that controls the viscosity. Since the ghost variables do not appear in the Chapman-Enskog expansion at Navier-Stokes order, changing the ghost variable relaxation times might be expected to leave the hydrodynamic variables unaffected.

However, on any finite lattice at a finite Reynolds number, there is a small but finite coupling between the hydrodynamic and ghost variables, as expressed by the Burnett and higher order terms in the Chapman-Enskog expansion [7, 34]. The strength of this coupling is set by the Knudsen numbers associated with the various modes, and thus by the coefficients $\tau_{\Pi}, \tau_{N}$, and $\tau_{J}$. For small Mach numbers, $O\left(\mathrm{Ma}^{-1}\right)$ timesteps are needed to reach a given macroscopic time, for instance a fixed multiple of an eddy turnover time. The timescale $\tau_{\Pi}$ associated with the viscous stress is scaled to be $O(\mathrm{Ma})$, and so correctly produces an $O(1)$ effect after $O\left(\mathrm{Ma}^{-1}\right)$ timesteps. If the coefficients $\tau_{N}$ and $\tau_{J}$ are not also scaled in proportion to the Mach number, as proposed in section 8 , they may cause an $O\left(\mathrm{Ma}^{-1}\right)$ divergence of the hydrodynamic variables away from the correct small Mach number limit though the accumulation of many numerically small, but Mach number independent, errors.

The necessary scaling of the relaxation times with Mach number may be achieved by introducing ghost Reynolds numbers as described in section 8. Using ghost Reynolds numbers that are small compared with the hydrodynamic Reynolds numbers, for instance $\mathrm{Re}=30000$ and $R_{N}=100$, to set the non-hydrodynamic relaxation times yields significant stability advantages over simulations performed with the BGK collision operator, without sacrificing the existence of an incompressible limit. Although many of the errors calculated in this paper are still numerically small, this is only because the solutions contain many lattice points across each feature. The errors become much larger for features close to the lattice scale, as in Fig. 7 , where the Knudsen numbers responsible for cross-coupling between hydrodynamic and ghost variables become much larger.

Finally, the analysis and numerical experiments in this paper are based on the common two dimensional nine speed lattice, but the conclusions should hold for general lattices whenever there is some coupling between hydrodynamic and ghost variables. In particular, the conclusions should hold for the larger lattices that use two or more different speeds per direction to simulate fully thermal gas dynamics with a spatially varying temperature. The number of hydrodynamic variables increases in these thermal lattice Boltzmann equations to include an independent heat flux vector, but the total number of variables usually increases even more to ensure isotropy. There is therefore additional scope for instabilities associated with the nonhydrodynamic modes to limit the accessible range of Reynold numbers, which may explain why thermal lattice Boltzmann equations have generally proved less successful than their isothermal predecessors [27].

\section{ACKNOWLEDGMENT}

Financial support from the Glasstone Benefaction at the University of Oxford is gratefully acknowledged.

\section{REFERENCES}

1. E. Anderson et al. LAPACK Users' Guide. SIAM, Philadelphia, 3rd edition, 1999. Available from http://www. netlib.org/lapack/lug/ lapack_lug.html. 
2. O. Behrend, R. Harris, and P. B. Warren. Hydrodynamic behavior of lattice Boltzmann and lattice Bhatnagar-Gross-Krook models. Phys. Rev. E, 50:4586-4595, 1994, doi:10.1103/PhysRevE.50.4586.

3. R. Benzi, S. Succi, and M. Vergassola. Turbulence modelling by nonhydrodynamic variables. Europhys. Lett., 13:727-732, 1990.

4. R. Benzi, S. Succi, and M. Vergassola. The lattice Boltzmann equation: theory and applications. Phys. Rept., 222:145-197, 1992.

5. P. L. Bhatnagar, E. P. Gross, and M. Krook. A model for collision process in gases. I. Small amplitude processes in charged and neutral one-component system. Phys. Rev., 94:511-525, 1954, doi:10.1103/PhysRev.94.511.

6. C. Cercignani. The Boltzmann Equation and its Applications. Springer-Verlag, New York, 1988.

7. S. Chapman and T. G. Cowling. The Mathematical Theory of Non-Uniform Gases. Cambridge University Press, Cambridge, 3rd edition, 1991.

8. S. Chen, H. Chen, D. O. Martínez, and W. H. Matthaeus. Lattice Boltzmann model for simulation of magnetohydrodynamics. Phys. Rev. Lett., 67:37763779, 1991, doi:10.1103/PhysRevLett.67.3776.

9. S. Chen and G. D. Doolen. Lattice Boltzmann method for fluid flows. Annu. Rev. Fluid Mech., 30:329-364, 1998.

10. S. P. Das, H. J. Bussemaker, and M. H. Ernst. Generalized hydrodynamics and dispersion relations in lattice gases. Phys. Rev. E, 48:245-255, 1993, doi:10.1103/PhysRevE.58.245.

11. P. J. Dellar. Bulk and shear viscosities in lattice Boltzmann equations. Phys. Rev. E, 64:031203, 2001, doi:10.1103/PhysRevE.64.031203.

12. P. J. Dellar. Nonhydrodynamic modes and a priori construction of shallow water lattice Boltzmann equations. Phys. Rev. E, 65:036309, 2002, doi:10.1103/PhysRevE.65.036309.

13. D. d'Humières. Generalized lattice-Boltzmann equations. In B. D. Shizgal and D. P. Weaver, editors, Rarefied Gas Dynamics: Theory and Simulations, volume 159 of Prog. Astronaut. Aeronaut., pages 450-458, Washington, D.C., 1992. AIAA.

14. D. d'Humières, I. Ginzburg, M. Krafczyk, P. Lallemand, and L.-S. Luo. Multiple-relaxation-time lattice Boltzmann models in three dimensions. Phil. Trans. R. Soc. Lond. A, 360:437-451, 2002, doi:10.1098/rsta.2001.0955.

15. M. Frigo and S. G. Johnson. FFTW: An adaptive software architecture for the FFT. In Proceedings of the IEEE International Conference on Acoustics, Speech, and Signal Processing, Seattle, 1998, volume 3, pages 1381-1384, Piscataway, NJ, 1998. IEEE Press. Available from http://www.fftw.org/.

16. U. Frisch, D. d'Humières, B. Hasslacher, P. Lallemand, Y. Pomeau, and J.-P. Rivet. Lattice gas hydrodynamics in two and three dimensions. Complex Sys., 1:649-707, 1987.

17. G. H. Golub and C. F. Van Loan. Matrix Computations. Johns Hopkins University Press, Baltimore, 3rd edition, 1996.

18. H. Grad. Principles of the kinetic theory of gases. In S. Flügge, editor, Thermodynamik der Gase, volume 12 of Handbuch der Physik, pages 205-294, Berlin, 1958. Springer-Verlag.

19. X. He, S. Chen, and G. D. Doolen. A novel thermal model of the lattice Boltzmann method in incompressible limit. J. Comput. Phys., 146:282-300, 1998, doi:10.1006/jcph.1998.6057.

20. X. He and L.-S. Luo. Lattice Boltzmann model for the incompressible Navier-Stokes equation. J. Statist. Phys., 88:927-944, 1997.

21. X. He and L.-S. Luo. Theory of the lattice Boltzmann method: From the Boltzmann equation to the lattice Boltzmann equation. Phys. Rev. E, 56:68116817, 1997, doi:10.1103/PhysRevE.56.6811.

22. X. He, X. Shan, and G. D. Doolen. Discrete Boltzmann equation model for nonideal gases. Phys. Rev. E, 57:R13-R16, 1998.

23. F. J. Higuera and J. Jiménez. Boltzmann approach to lattice gas simulations. Europhys. Lett., 9:663-668, 1989.

24. F. J. Higuera, S. Succi, and R. Benzi. Lattice gas dynamics with enhanced collisions. Europhys. Lett., 9:345-349, 1989.

25. P. Lallemand and L.-S. Luo. Theory of the lattice Boltzmann method: Dispersion, dissipation, isotropy, Galilean invariance, and stability. Phys. Rev. E, 61:6546-6562, 2000

26. L.-S. Luo, H. Chen, S. Chen, G. D. Doolen, and Y.-C. Lee. Generalized hydrodynamic transport in lattice-gas automata. Phys. Rev. A, 43:7097-7100, 1991, doi:10.1103/PhysRevA.43.7097.

27. G. R. McNamara, A. L. Garcia, and B. J. Alder. Stabilization of thermal lattice Boltzmann models. J. Statist. Phys., 81:395-408, 1995.

28. M. L. Minion and D. L. Brown. Performance of under-resolved two-dimensional incompressible flow simulations, II. J. Comput. Phys., 138:734-765, 1997, doi:10.1006/jcph.1997.5843.

29. Y. H. Qian, D. d'Humières, and P. Lallemand. Lattice BGK models for the Navier-Stokes equation. Europhys. Lett., 17:479-484, 1992.

30. P. A. Skordos. Initial and boundary conditions for the lattice Boltzmann method. Phys. Rev. E, 48:4823-4842, 1993, doi:10.1103/PhysRevE.48.4823.

31. J. D. Sterling and S. Chen. Stability analysis of lattice Boltzmann methods. J. Comput. Phys., 123:196-206, 1996, doi:10.1006/jcph.1996.0016.

32. S. Succi. The Lattice Boltzmann Equation: For Fluid Dynamics and Beyond. Oxford University Press, Oxford, 2001.

33. S. Succi, I. V. Karlin, and H. Chen. Role of the H theorem in lattice Boltzmann hydrodynamic simulations. Rev. Mod. Phys., 74:1203-1220, 2002, doi:10.1103/RevModPhys.74.1203.

34. G. E. Uhlenbeck and G. W. Ford. Lectures in Statistical Mechanics, volume 1 of Lectures in Applied Mathematics. American Mathematical Society, Providence, 1963

35. Q. S. Zou, S. L. Hou, S. Y. Chen, and G. D. Doolen. An improved incompressible lattice Boltzmann model for time-independent flows. J. Statist. Phys., 81:35-48, 1995. 